\title{
Controlled Flexible Coordination in Tripodal Iron(II) Phosphane Complexes: Effects on Reactivity
}

Anette Petuker, ${ }^{a}$ Klaus Merz, $^{a}$ Christian Merten ${ }^{* b}$ and Ulf-Peter Apfel ${ }^{* a}$

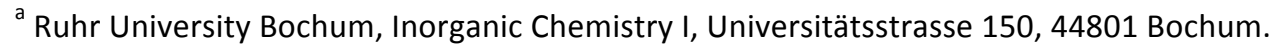

${ }^{b}$ Ruhr University Bochum, Physical Organic Chemistry, Universitätsstrasse 150, 44801 Bochum. 


\section{Table of contents}

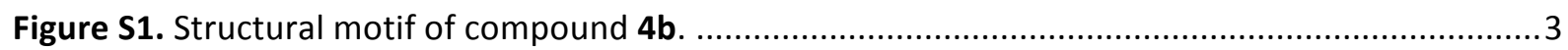

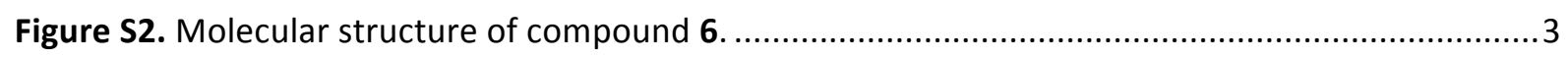

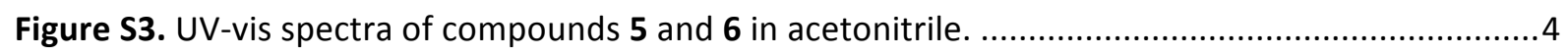

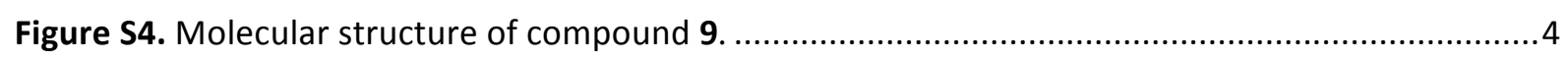

Figure S5. UV-vis spectra of compounds $\mathbf{4 a}$ and $\mathbf{7}$ in different solvents and various temperatures ......5

Figure S6. UV-vis spectra of compounds $\mathbf{4 a}$ and $\mathbf{7}$ in different solvents and various temperatures ......6

Figure S7. UV-vis spectra of compounds $\mathbf{4 a}$ and $\mathbf{7}$ in different solvents and various temperatures .......7

Figure S8. Mössbauer spectrum of 7, 8 and an equilibrium mixture of complexes $\mathbf{7}$ and 8 ................8

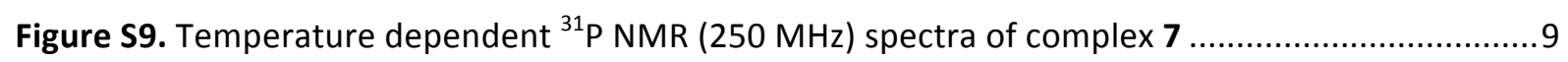

Figure S10. Temperature dependent changes of the magnetic moment of complex $\mathbf{7}$ dissolved in acetonitrile.

Figure S11. Van't Hoff plot for the equilibrium reaction of complex $\mathbf{7}$ and $\mathbf{8}$ in acetonitrile from UV-vis

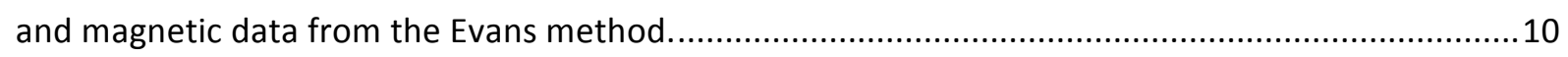

Table S1. Thermodynamic parameters for the equilibrium reaction of complex $\mathbf{7}$ and 8 ..................10

Figure S12. UV-vis spectrum of complex $\mathbf{9}$ in acetonitrile at various temperatures ...........................11

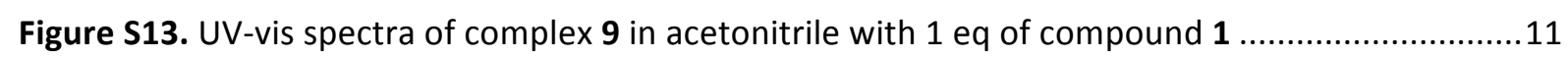

Figure S14. Van't Hoff plot for the rearrangement of complex 9 in acetonitrile................................12

Table S2. Thermodynamic parameters for the rearrangement of complex 9 in acetonitrile ...............12

Figure S15. UV-vis spectroscopic changes of an equilibrium mixture of complexes $\mathbf{7}$ and $\mathbf{8}$ in

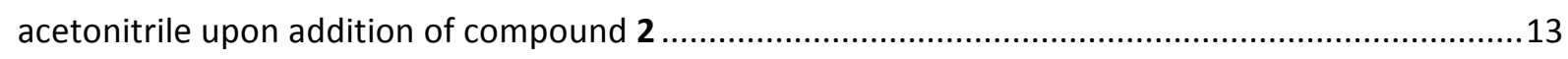

Figure S16. UV-vis spectroscopic changes of an equilibrium mixture of complexes $\mathbf{7}$ and $\mathbf{8}$ in

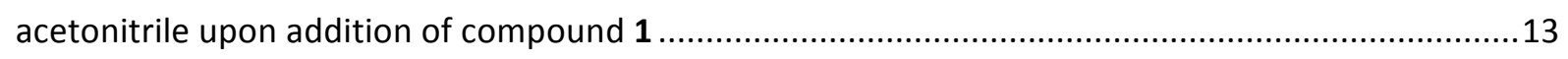

Figure S17. Oxidative decomposition of $\mathbf{4 a}$ and $\mathbf{7}$ under oxygen exposure .....................................14

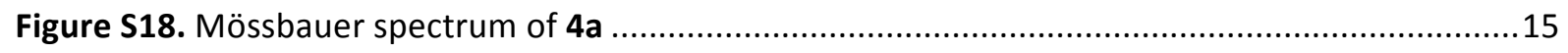

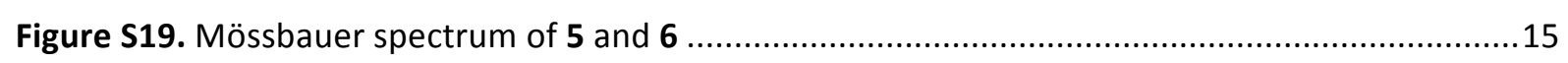

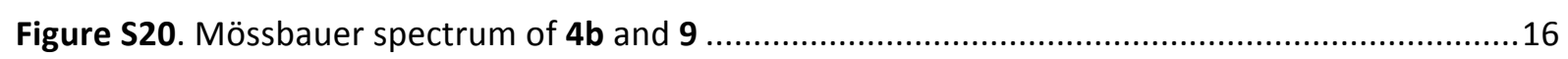

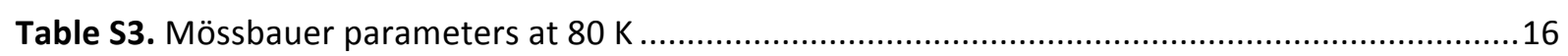

Figure S21. ${ }^{1} \mathrm{H}$ (top) and ${ }^{13} \mathrm{C}$ NMR spectra (bottom) of 1-phenylethan-1-ol.........................................17

Figure S22. GC-trace (top) and MS- spectrum (bottom) of 1-phenylethan-1-ol.....................................18

Table S4. Crystal Data and Refinement Details for Compounds 4a, 4b, 5, 6 ....................................19

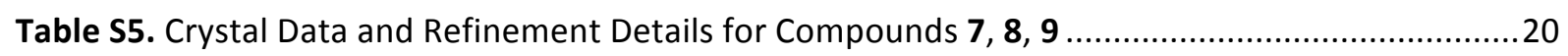

Table S6. Relative energy differences of the calculated conformers of tetrahedral $4 a$ and 7 ...........21 


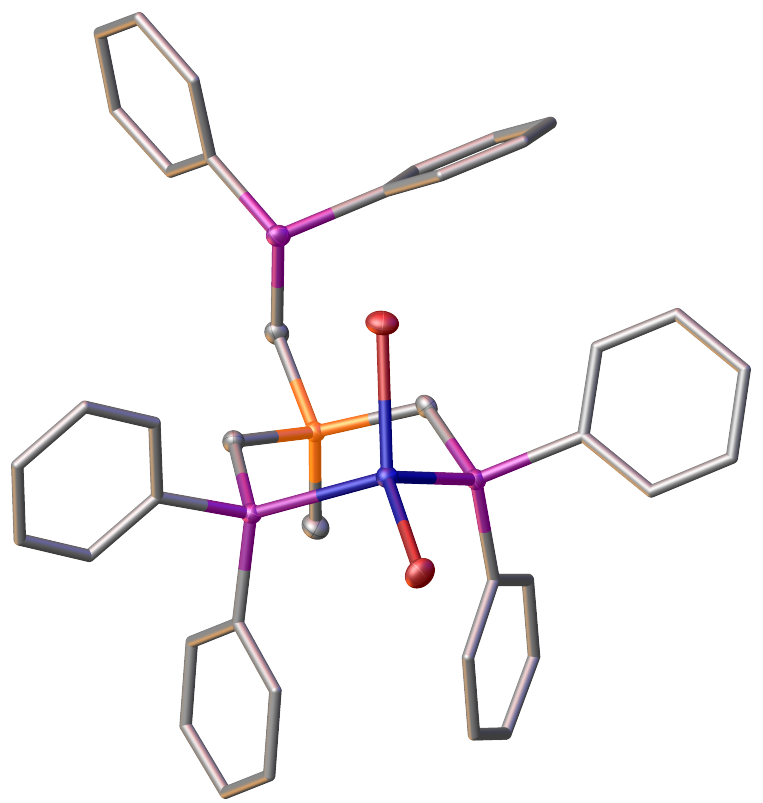

Figure S1. Structural motif of compound $\mathbf{4 b}$ with thermal ellipsoids at the $50 \%$ probability level (hydrogen atoms and thermal ellipsoids of the phenyl groups were omitted for clarity).

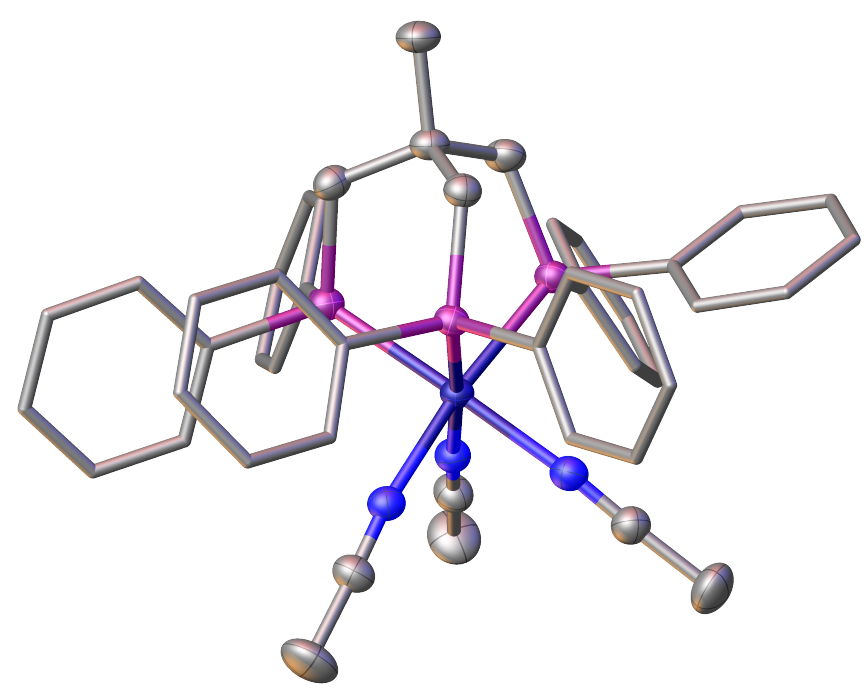

Figure S2. Molecular structure of compound 6 with thermal ellipsoids at the 50\% probability level (hydrogen atoms and thermal ellipsoids of the phenyl groups were omitted for clarity). 


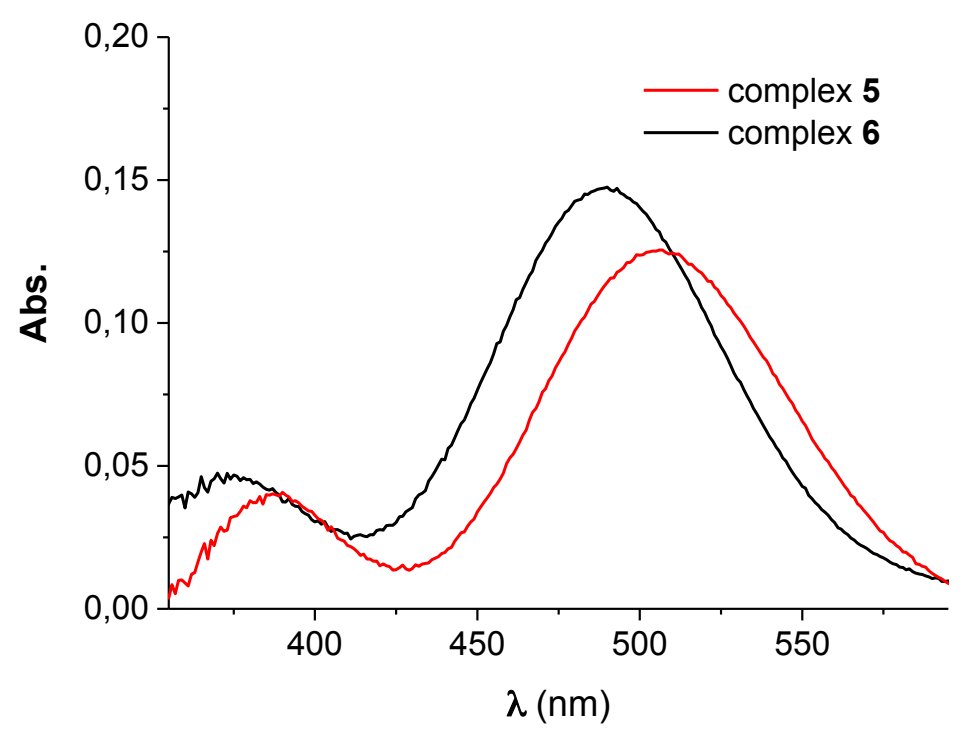

Figure S3. UV-vis spectra of compounds $\mathbf{5}$ and $\mathbf{6}$ in acetonitrile.

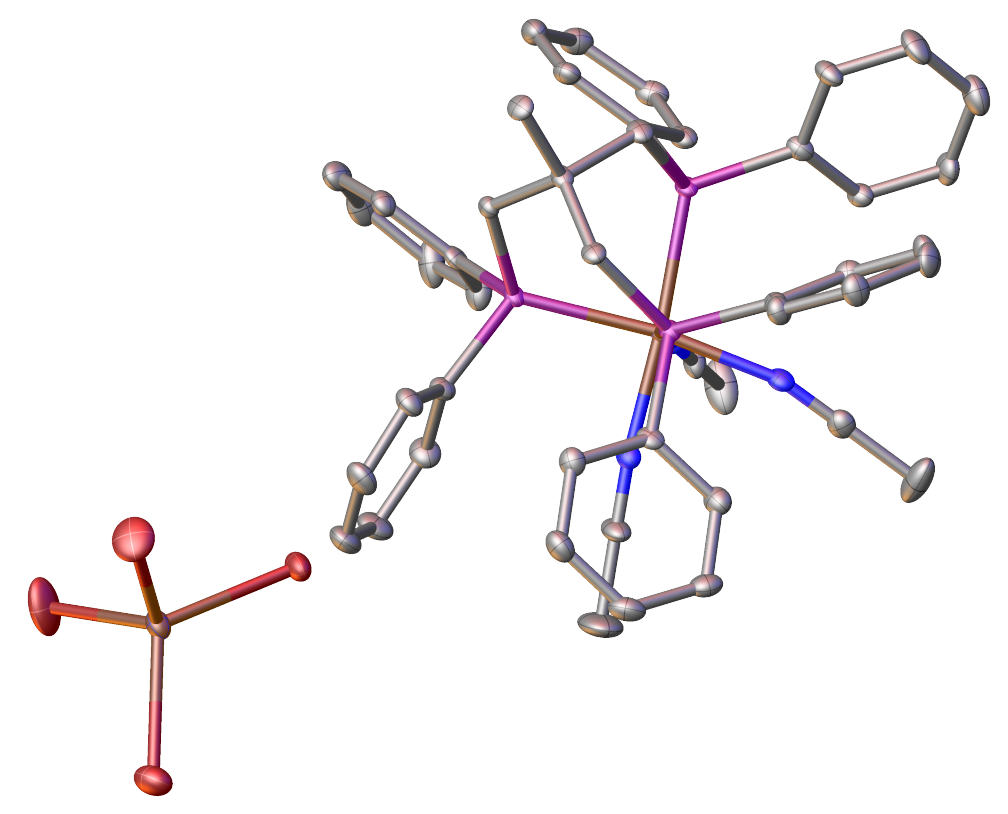

Figure S4. Molecular structure of compound 9 with thermal ellipsoids at the $50 \%$ probability level (hydrogen atoms and thermal ellipsoids of the phenyl groups were omitted for clarity). 

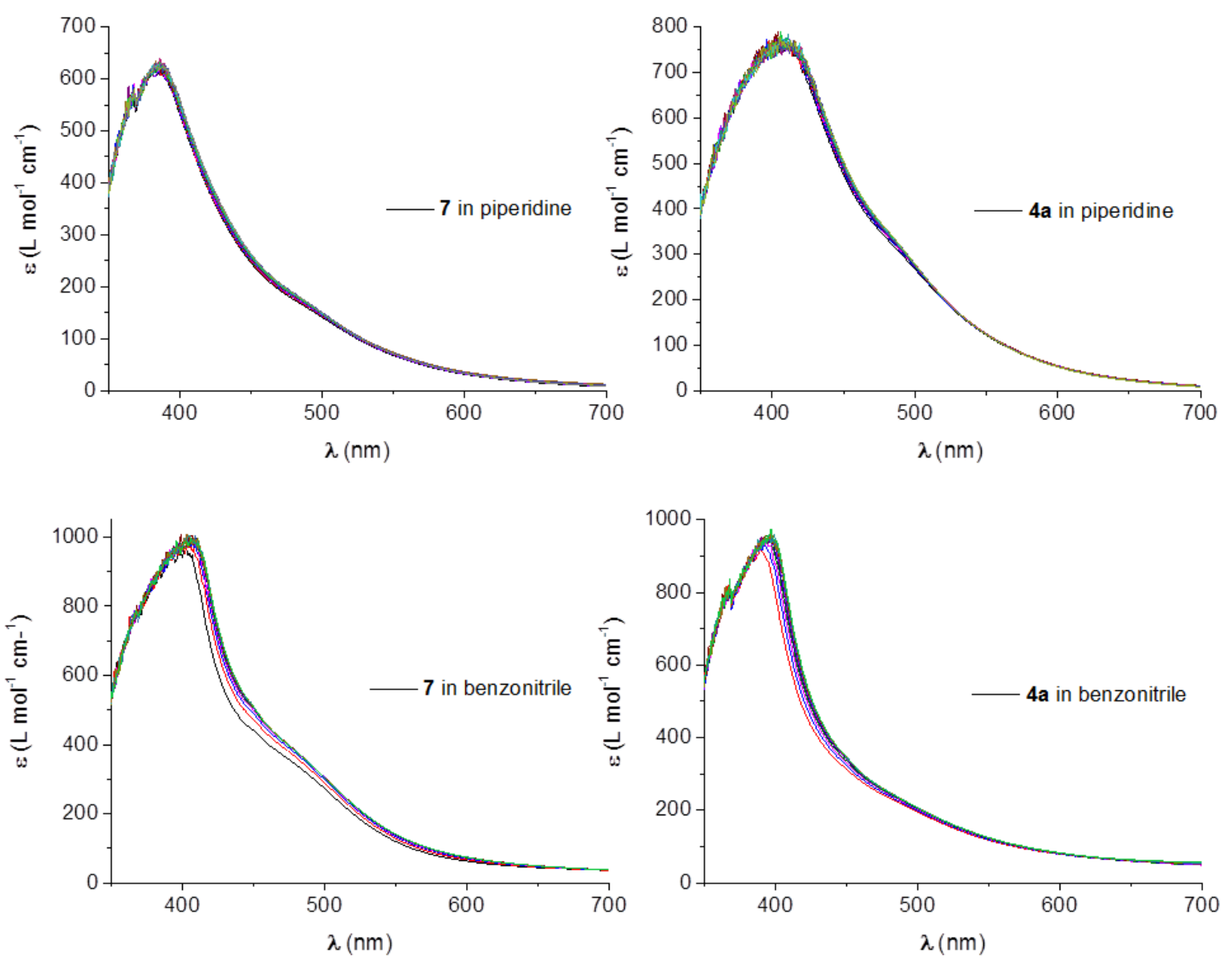

Figure S5. UV-vis spectra of compounds $\mathbf{4 a}$ and $\mathbf{7}$ in different solvents and various temperatures (20 to $70^{\circ} \mathrm{C}$ ). 

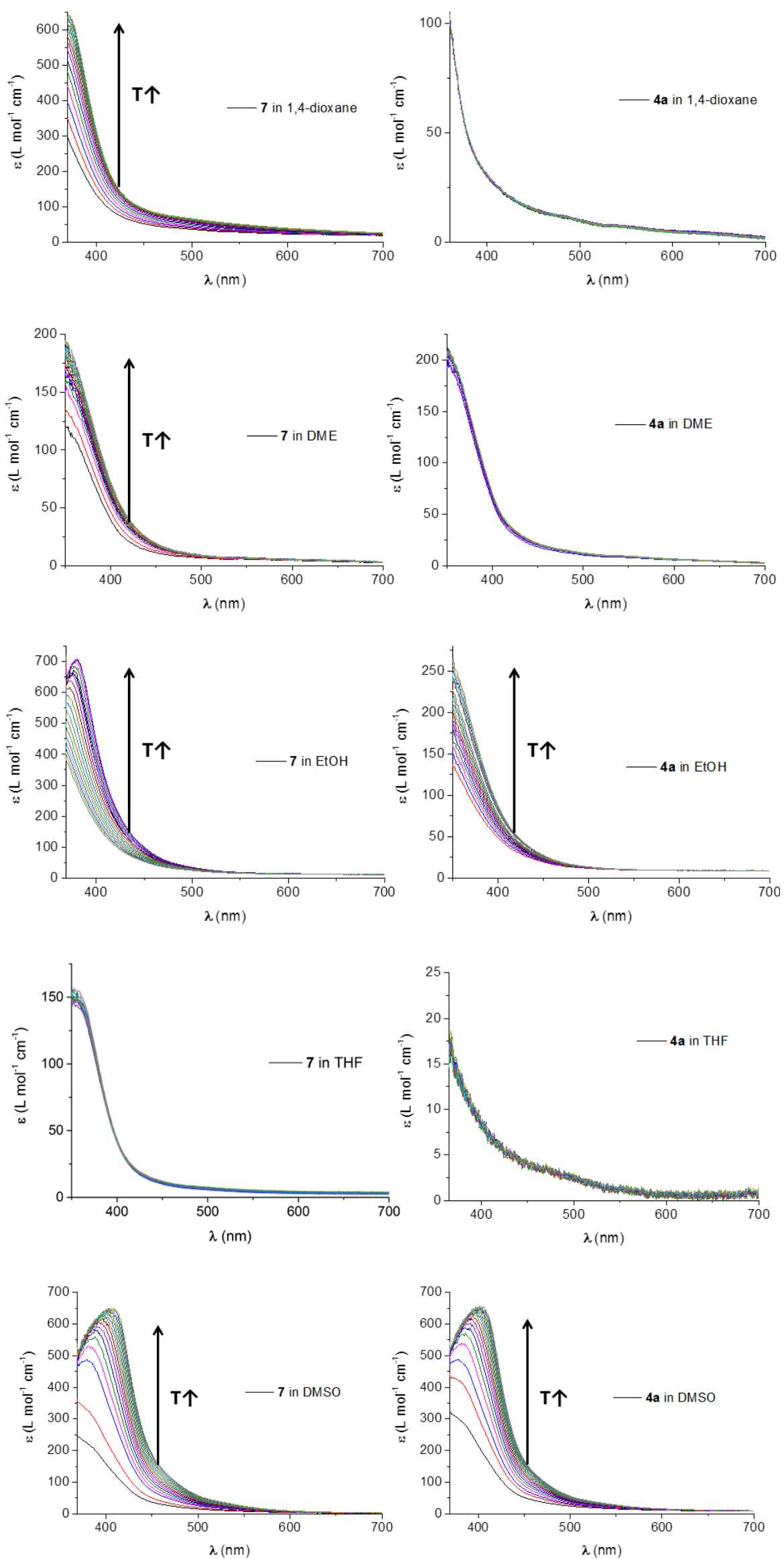

Figure S6. UV-vis spectra of compounds $\mathbf{4 a}$ and $\mathbf{7}$ in different solvents and various temperatures (20 to $70^{\circ} \mathrm{C}$ ). 

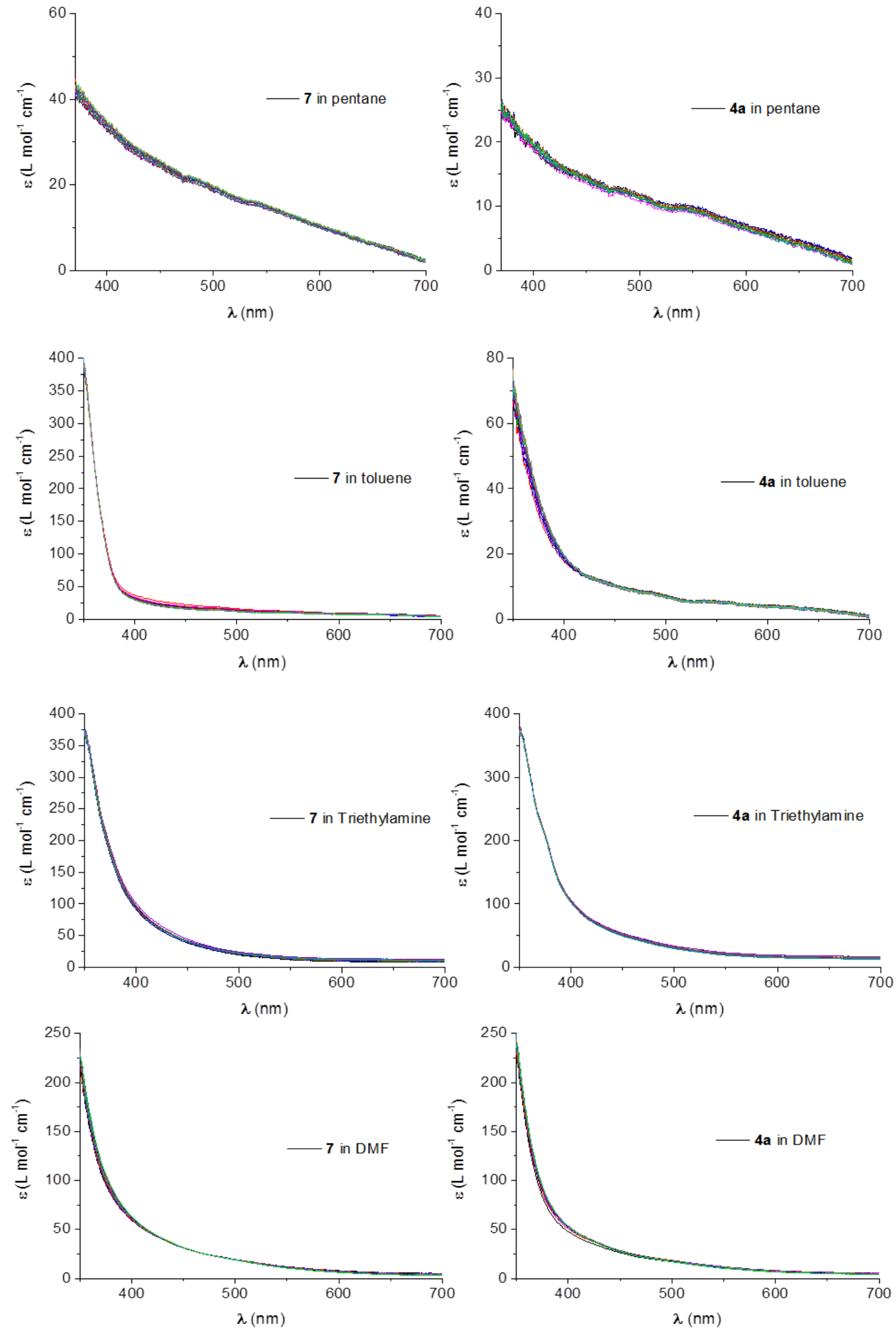

Figure S7. UV-vis spectra of compounds $\mathbf{4 a}$ and $\mathbf{7}$ in different solvents and various temperatures (20 to $70^{\circ} \mathrm{C}$ ). 

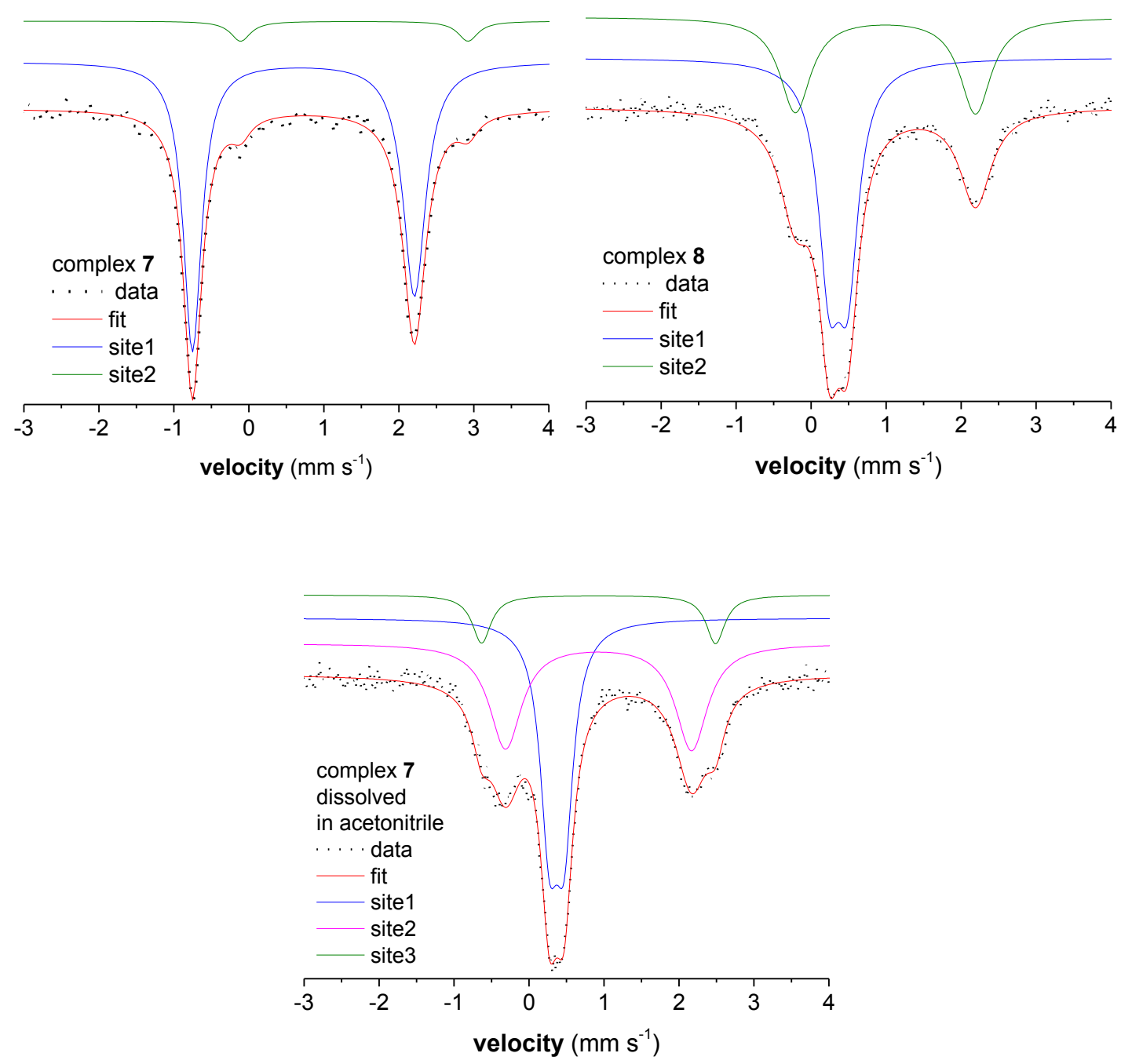

Figure 58. Mössbauer spectrum of $\mathbf{7}$ (crystalline sample, top), $\mathbf{8}$ (crystalline sample, middle) and an equilibrium mixture of complexes $\mathbf{7}$ and $\mathbf{8}$ (in acetonitrile, bottom) at $80 \mathrm{~K}$. The equilibrium mixture was obtained by dissolving $\mathbf{7}$ in acetonitrile. Once everything was dissolved, the solution was frozen in liquid nitrogen and used as is for Mössbauer measurements. For compounds $\mathbf{7}$ and complex $\mathbf{8}$ dissolved in acetonitrile, the area ratio of site 1 to site 2 is roughly 45:55 which differs from the expected 33:66 ratio in [(Triphos) $\left.\mathrm{Fe}\left(\mathrm{CH}_{3} \mathrm{CN}\right)_{3}\right]\left(\mathrm{Fe}_{2} \mathrm{Cl}_{6}\right)$. The discrepancy can be explained by different spin relaxation of the $\left[(\text { Triphos }) \mathrm{Fe}\left(\mathrm{CH}_{3} \mathrm{CN}\right)_{3}\right]^{+}$cation and the $\left[\mathrm{Fe}_{2} \mathrm{Cl}_{6}\right]^{2-}$ anion. A small paramagnetic $\mathrm{Fe}$ impurity can be observed in the spectrum of compound 7 . 


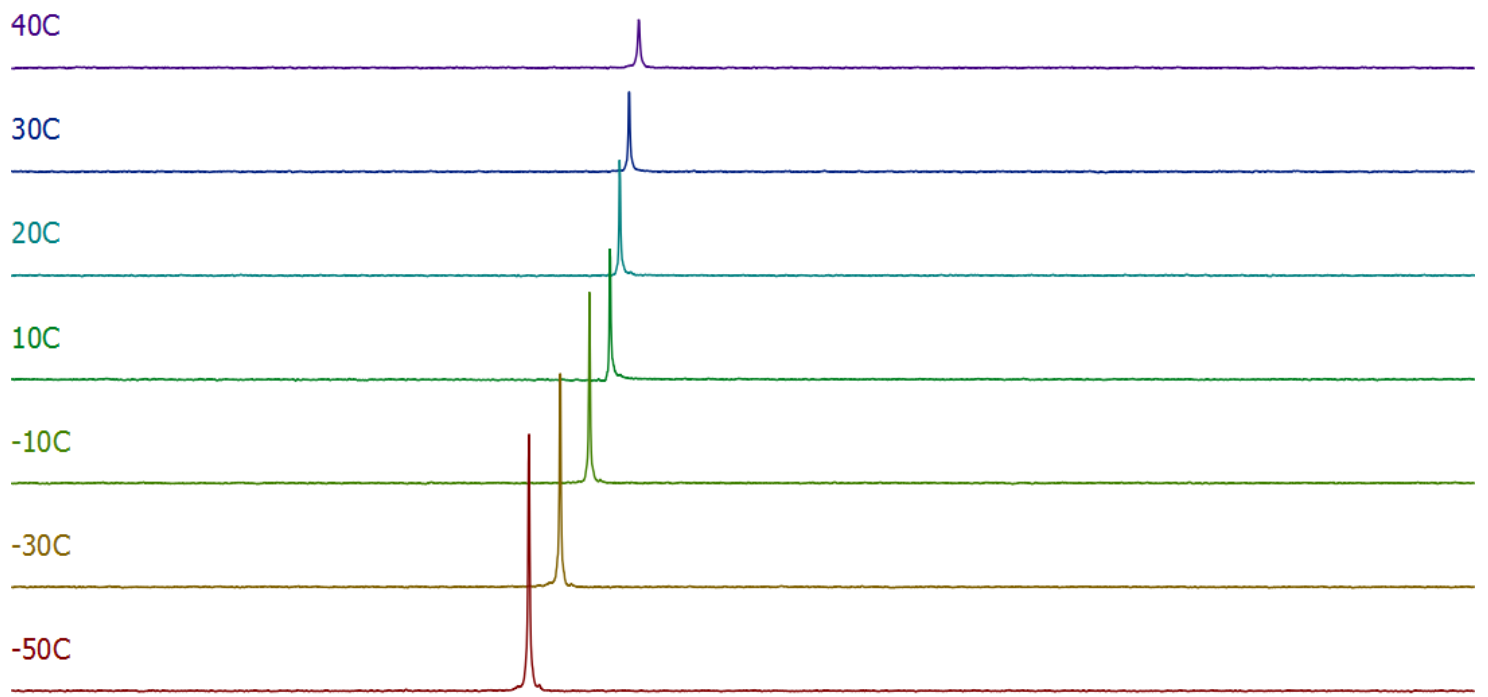

$\begin{array}{lllllllllllllllllllllllllll}43 & 42 & 41 & 40 & 39 & 38 & 37 & 36 & 35 & 34 & 33 & 32 & \begin{array}{c}31 \\ \mathrm{f} 1(\mathrm{ppm})\end{array} & 30 & 29 & 27 & 26 & 25 & 24 & 23 & 22 & 21 & 20 & 19 & 18 & 1 \text { : }\end{array}$

Figure S9. Temperature dependent ${ }^{31} \mathrm{P}$ NMR $(250 \mathrm{MHz})$ spectra of complex 7 in deuterated acetonitrile.

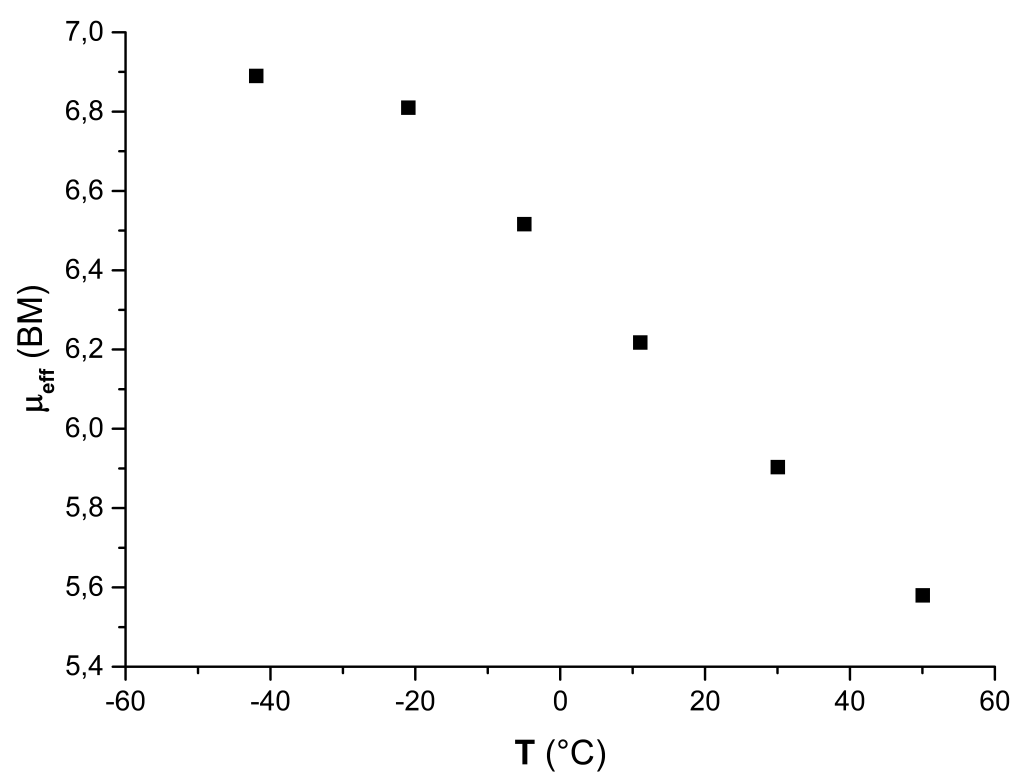

Figure S10. Temperature dependent changes of the magnetic moment of complex 7 dissolved in acetonitrile. 
3

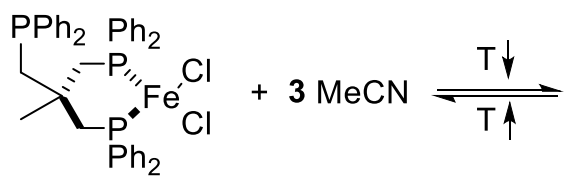

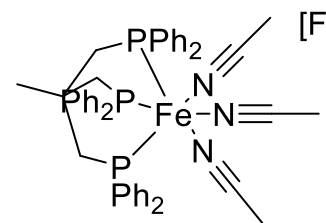

$\left[\mathrm{Fe}_{2} \mathrm{Cl}_{6}\right]$<smiles>CC(CP)(CPc1ccccc1)Cc1ccccc1</smiles>

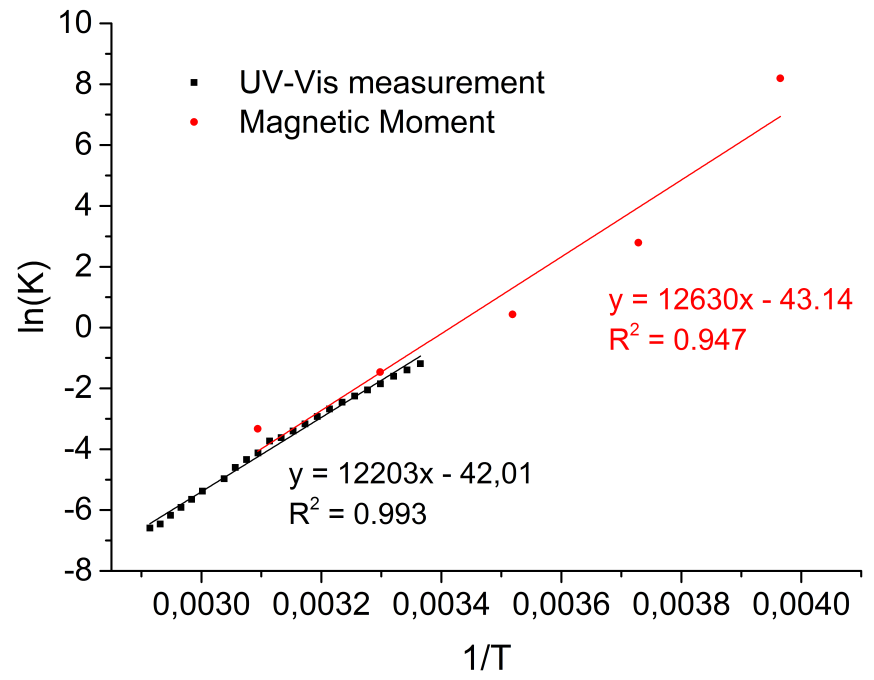

Figure S11. Van't Hoff plot for the equilibrium reaction of complex $\mathbf{7}$ and $\mathbf{8}$ in acetonitrile from UV-vis and magnetic data from the Evans method.

Table S1. Thermodynamic parameters for the equilibrium reaction of complex $\mathbf{7}$ and $\mathbf{8}$ in acetonitrile.

UV-vis measurement

\begin{tabular}{cc}
\hline $\mathrm{T}[\mathrm{K}]$ & $\mathrm{K}$ \\
\hline 293,15 & 0.39788004 \\
295,15 & 0.37519876 \\
297,15 & 0.30426768 \\
299,15 & 0.24698742 \\
301,15 & 0.20088746 \\
303,15 & 0.15728026 \\
305,15 & 0.12804447 \\
307,15 & 0.10481725 \\
309,15 & $0 ., 08578584$ \\
311,15 & 0.06874762 \\
313,15 & 0.05346117 \\
315,15 & 0.04189825 \\
317,15 & 0.03321905 \\
319,15 & 0.0267538 \\
321,15 & 0.02390934 \\
323,15 & 0.01622844 \\
325,15 & 0.01304995 \\
327,15 & 0.01006286 \\
329,15 & 0.00692665 \\
333,15 & 0.00460459 \\
335,15 & 0.00352163 \\
337,15 & 0.00269797 \\
339,15 & 0.00208780 \\
341,15 & 0.00156292 \\
343,15 & 0.00137159 \\
\hline &
\end{tabular}

\section{Magnetic Moment}

$\begin{array}{cc}\mathrm{T}[\mathrm{K}] & \mathrm{K} \\ 323,2 & 0,035838456 \\ 303,2 & 0,231455529 \\ 284,2 & 1,547912304 \\ 268,2 & 16,33986626 \\ 252,2 & 3613,712505\end{array}$

UV-vis measurement

\begin{tabular}{cc}
\hline$\Delta \mathrm{H}_{298 \mathrm{~K}}[\mathrm{~kJ} / \mathrm{mol}]$ & $\Delta \mathrm{S}_{298 \mathrm{~K}}[\mathrm{~J} / \mathrm{mol} \cdot \mathrm{K}]$ \\
\hline$-101.46( \pm 1.82)$ & $-349.29( \pm 4.71)$ \\
\hline
\end{tabular}

\section{Magnetic Moment}

\begin{tabular}{cc}
\hline$\Delta \mathrm{H}_{298 \mathrm{~K}}[\mathrm{~kJ} / \mathrm{mol}]$ & $\Delta \mathrm{S}_{298 \mathrm{~K}}[\mathrm{~J} / \mathrm{mol} \cdot \mathrm{K}]$ \\
\hline$-105.02( \pm 14.3)$ & $-357.52( \pm 50.55)$ \\
\hline
\end{tabular}

$$
\begin{gathered}
\mathrm{K}_{\mathrm{MeCN}}=\frac{\left([\text { Triphos }]^{2}[\mathbf{8}]\right)}{[7]^{3}} \\
\ln (\mathrm{K})=\frac{\Delta \mathrm{H}}{\mathrm{RT}}+\frac{\Delta \mathrm{S}}{\mathrm{R}}
\end{gathered}
$$




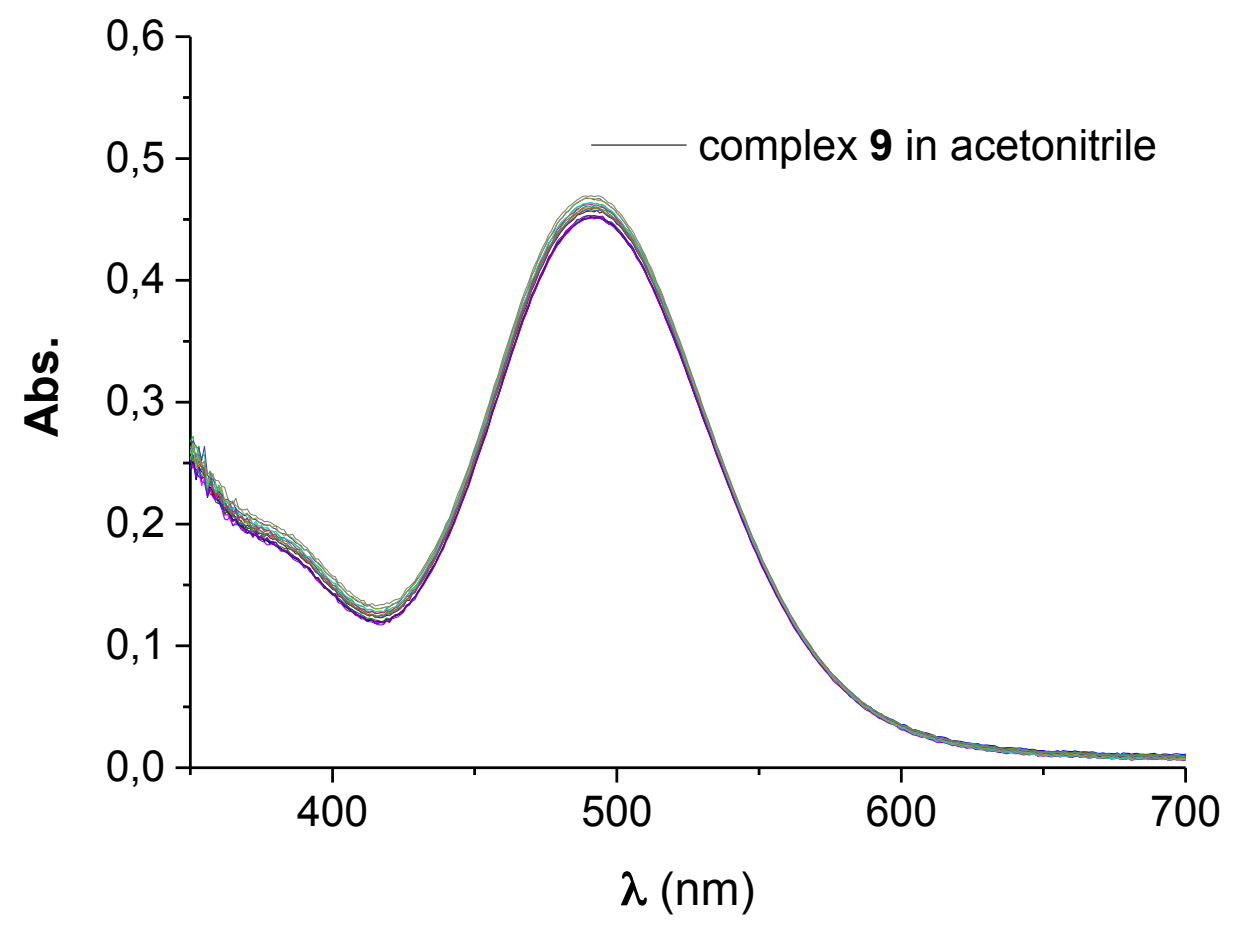

Figure S12. UV-vis spectrum of complex 9 in acetonitrile at various temperatures (20 to $70^{\circ} \mathrm{C}$ ).

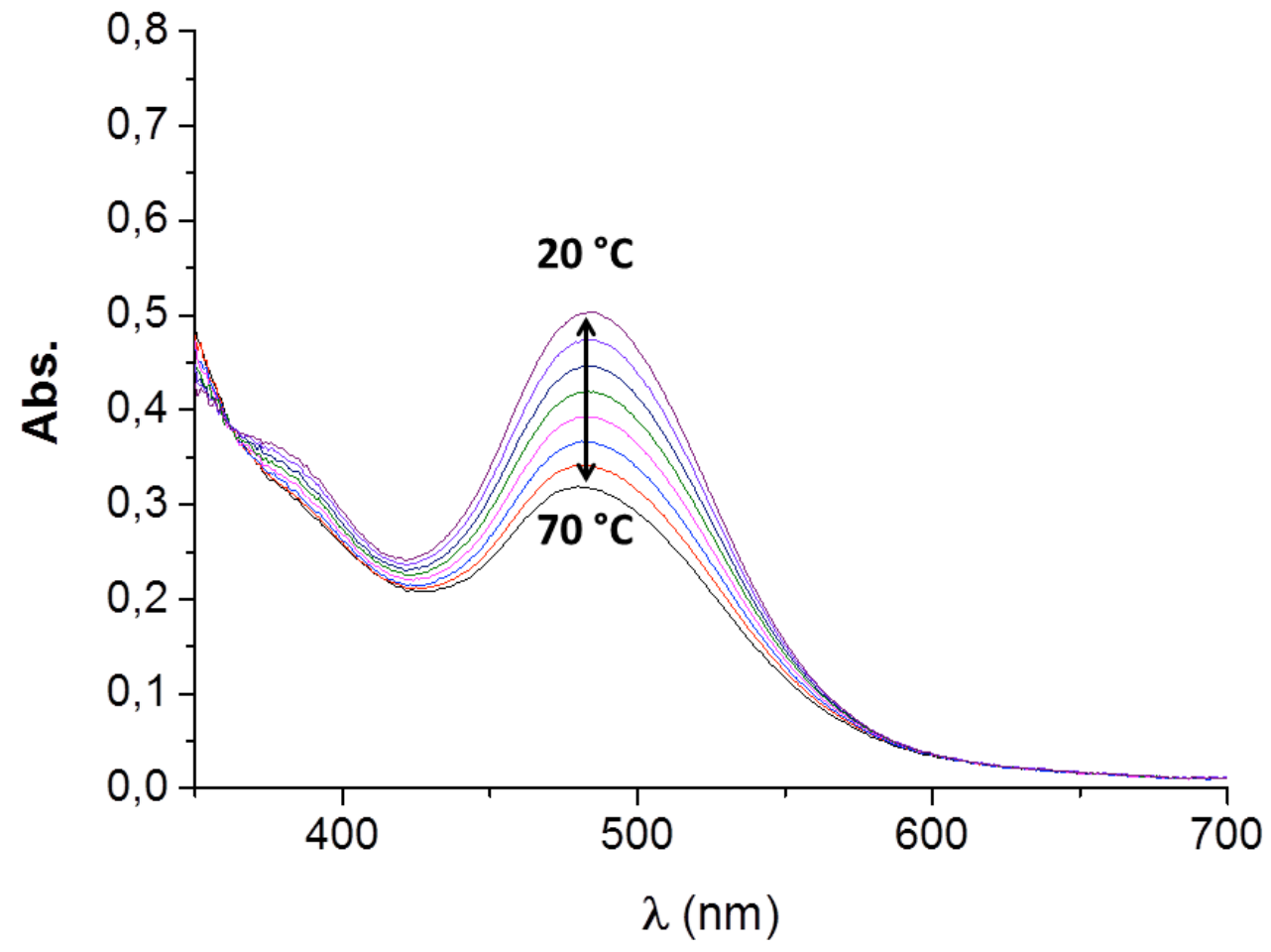

Figure S13. UV-vis spectra of complex 9 in acetonitrile with 1 eq of compound 1. 

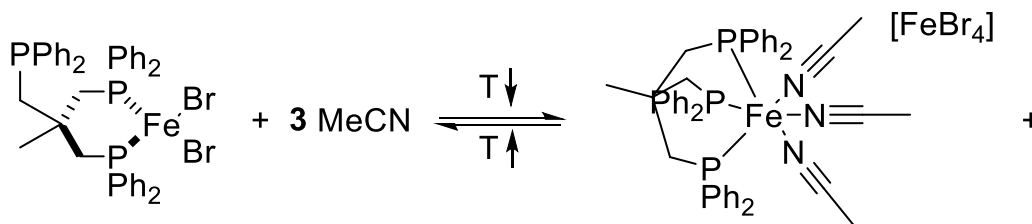<smiles>CC(CP)(CPP)CP(CP)c1ccccc1</smiles>

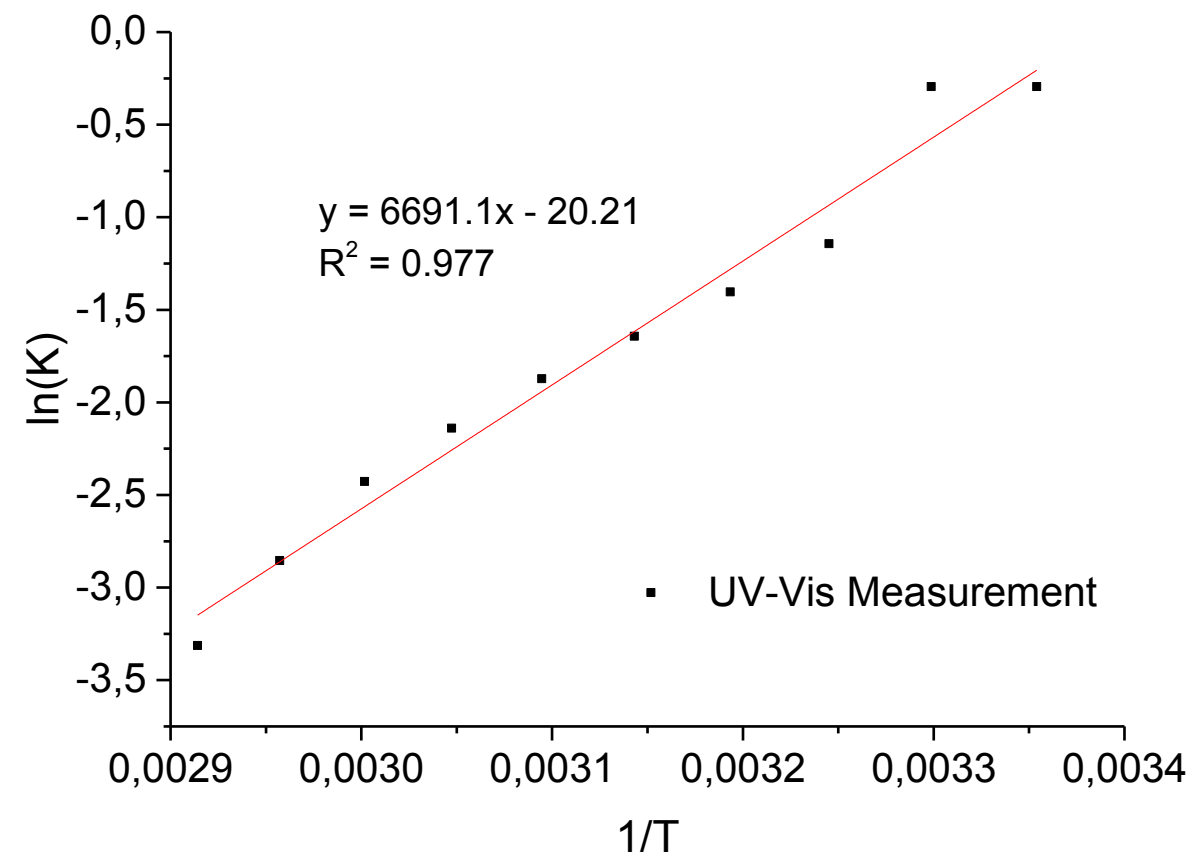

Figure S14. Van't Hoff plot for the rearrangement of complex 9 in acetonitrile.

Table S2. Thermodynamic parameters for the rearrangement of complex $\mathbf{9}$ in acetonitrile.

\begin{tabular}{cc}
\hline$T[K]$ & $K$ \\
\hline 298,15 & 0,744558798 \\
303,15 & 0,744558798 \\
308,15 & 0,318701011 \\
313,15 & 0,245845487 \\
318,15 & 0,193371577 \\
323,15 & 0,153743831 \\
328,15 & 0,117687775 \\
333,15 & 0,088254656 \\
338,15 & 0,057575155 \\
343,15 & 0,036405805
\end{tabular}

\begin{tabular}{cc}
\hline$\Delta \mathrm{H}_{298 \mathrm{~K}}[\mathrm{~kJ} / \mathrm{mol}]$ & $\Delta \mathrm{S}_{298 \mathrm{~K}}[\mathrm{~J} / \mathrm{mol} \cdot \mathrm{K}]$ \\
\hline$-55.63( \pm 2.21)$ & $-168.03( \pm 8.51)$ \\
\hline
\end{tabular}

$$
\begin{gathered}
\mathrm{K}_{\mathrm{MeCN}}=\frac{([\text { Triphos }][\mathbf{9}])}{[\mathbf{4 b}]^{2}} \\
\ln (\mathrm{K})=\frac{\Delta \mathrm{H}}{\mathrm{RT}}+\frac{\Delta \mathrm{S}}{\mathrm{R}}
\end{gathered}
$$




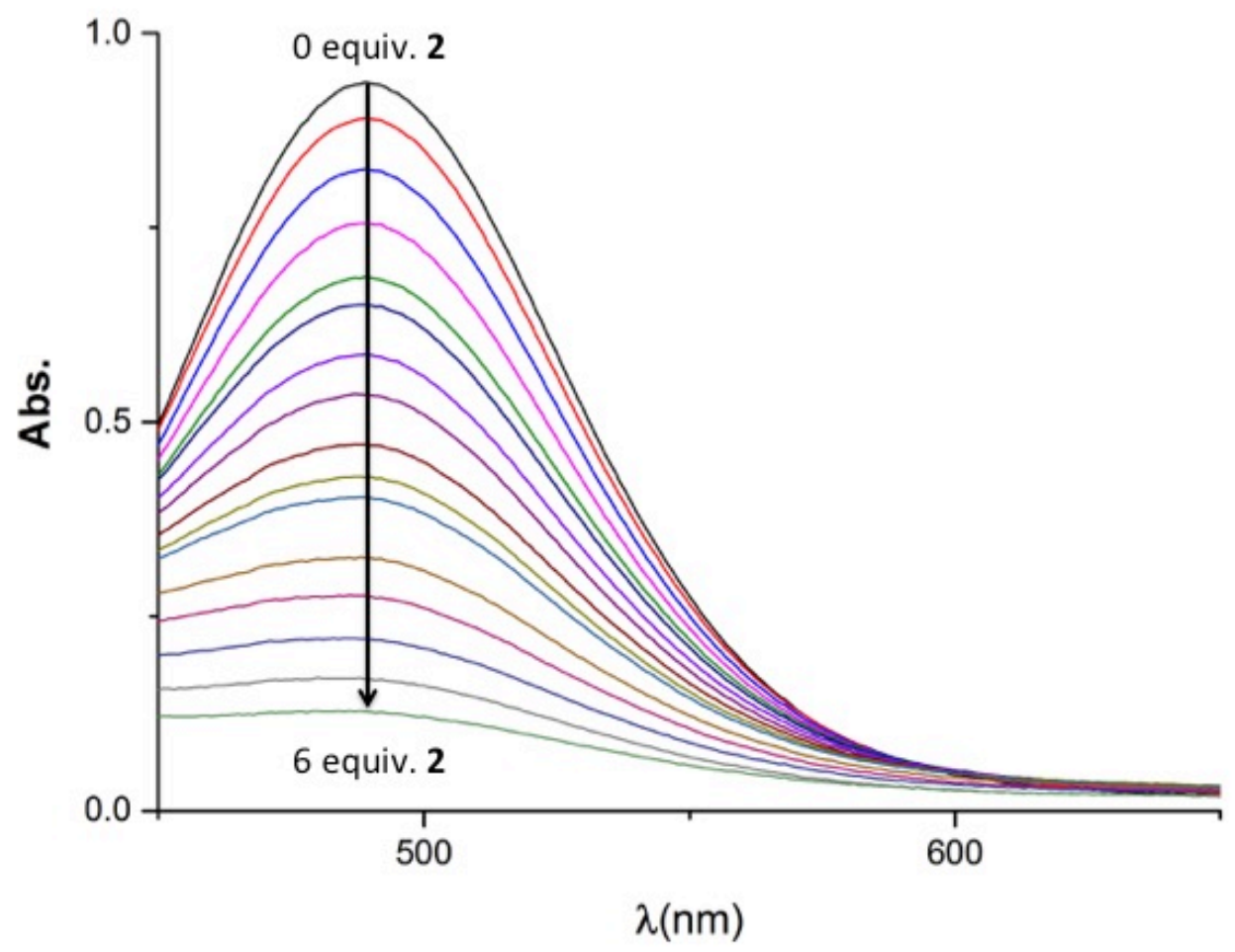

Figure S15. UV-vis spectroscopic changes of an equilibrium mixture of complexes $\mathbf{7}$ and $\mathbf{8}$ in acetonitrile upon addition of compound 2.

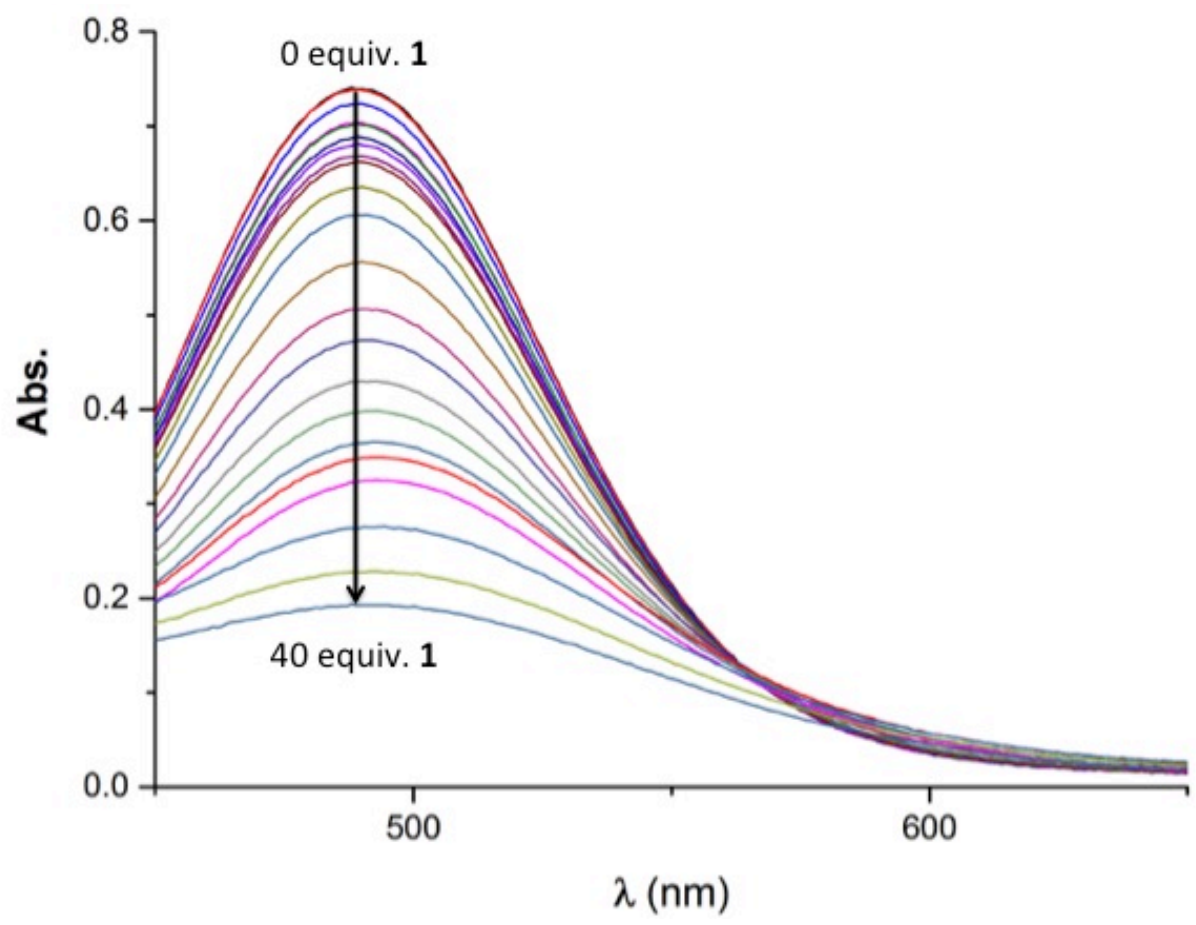

Figure S16. UV-vis spectroscopic changes of an equilibrium mixture of complexes $\mathbf{7}$ and $\mathbf{8}$ in acetonitrile upon addition of compound 1. 

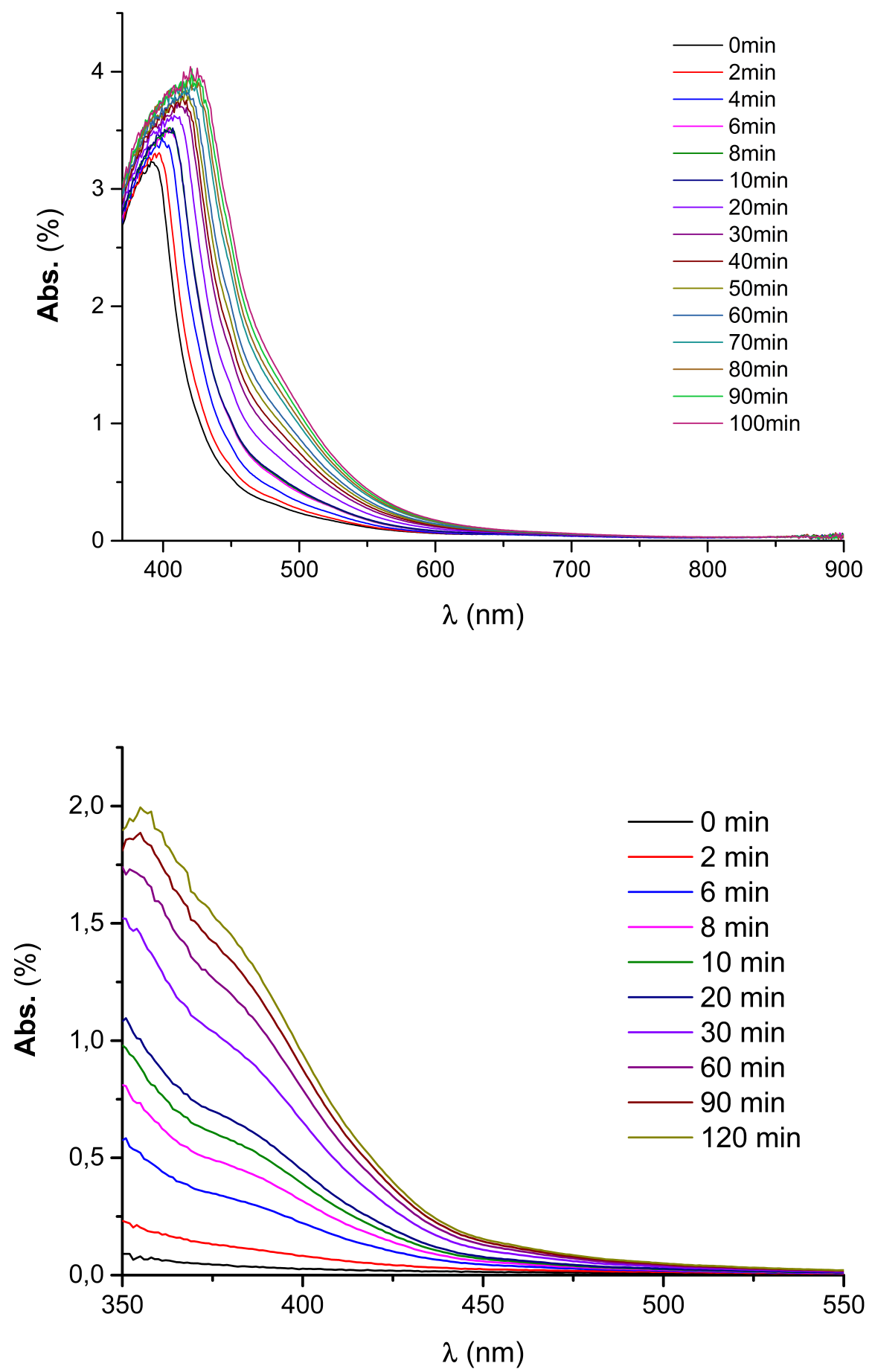

Figure S17. Oxidative decomposition of $4 \mathbf{a}$ and $\mathbf{7}$ under oxygen exposure. 


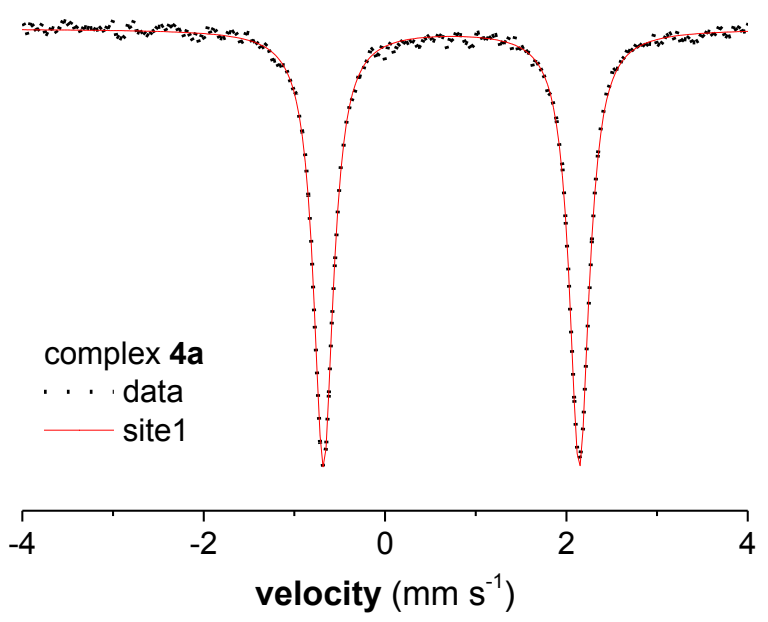

Figure S18. Mössbauer spectrum of 4a (solid sample, $80 \mathrm{~K}$ ).
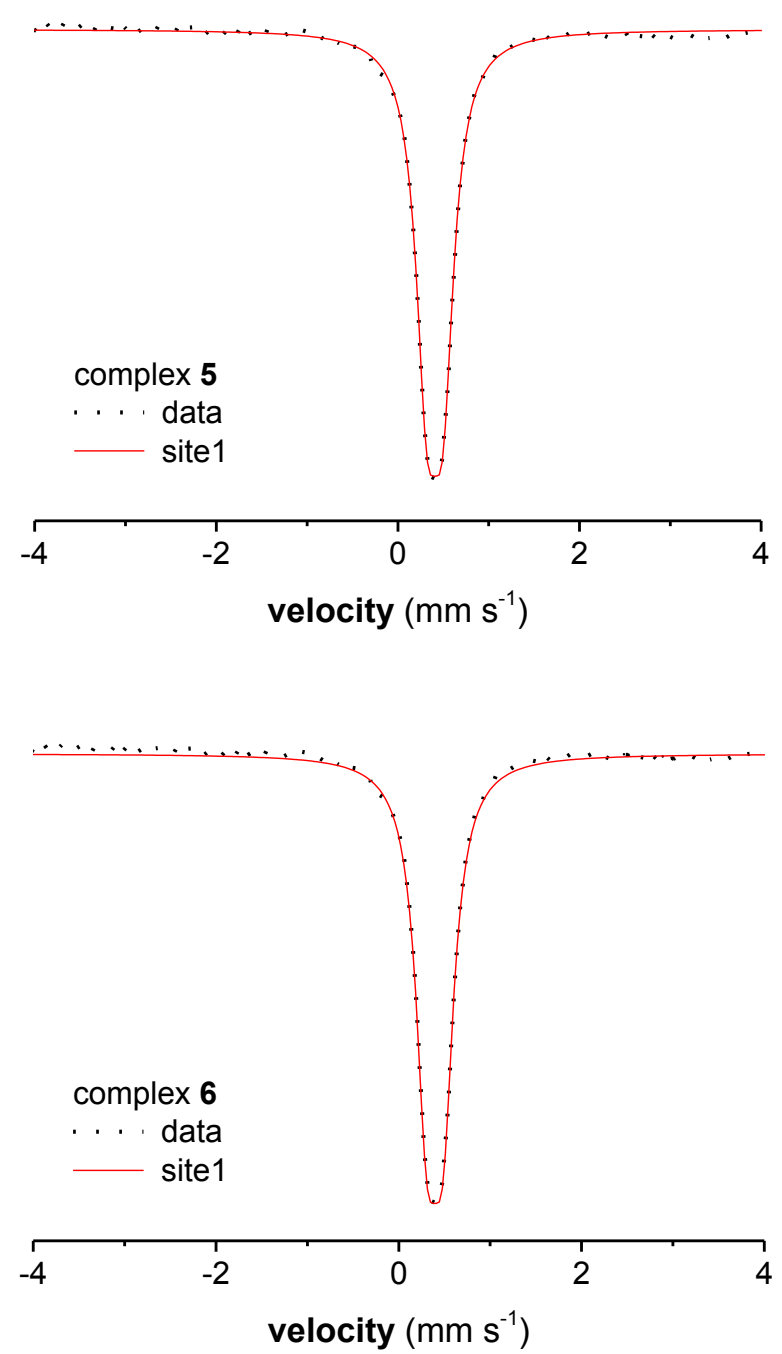

Figure S19. Mössbauer spectrum of $\mathbf{5}$ (top) and $\mathbf{6}$ (bottom) (solid samples, $80 \mathrm{~K}$ ). 

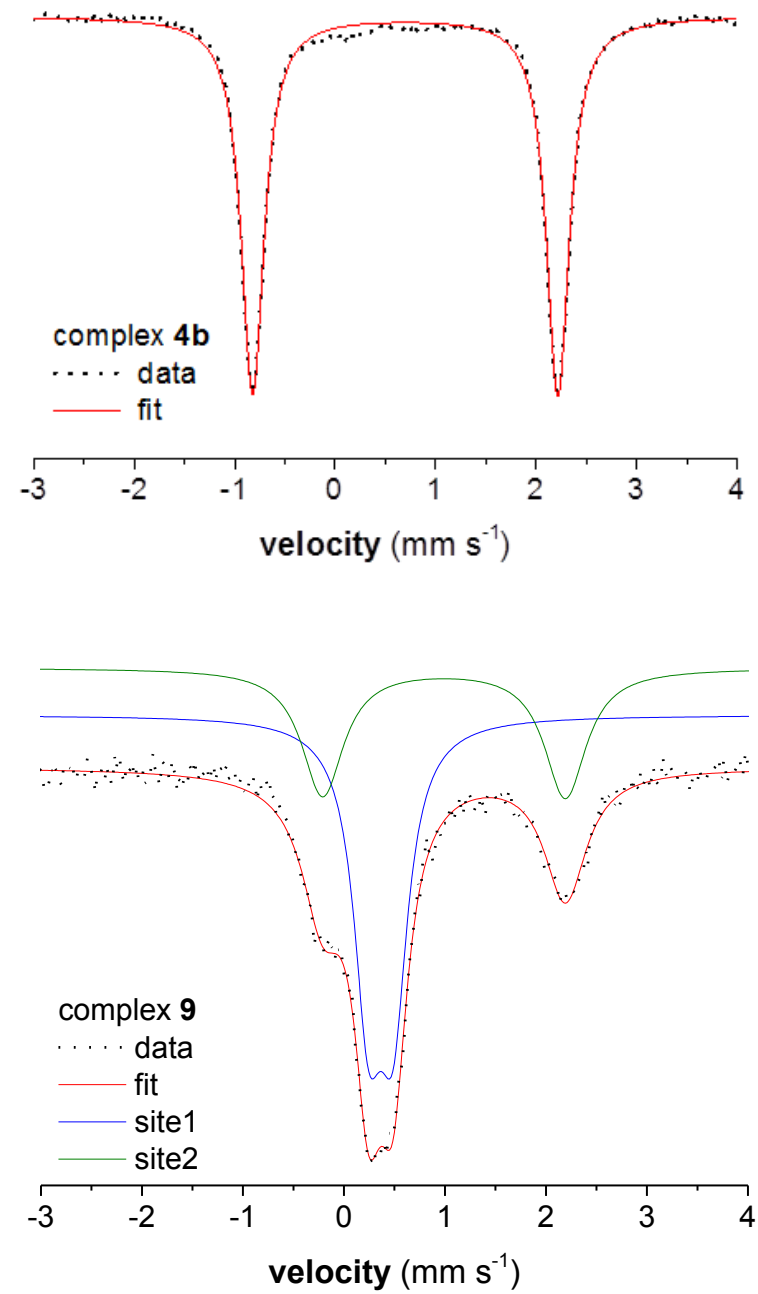

Figure S20. Mössbauer spectrum of $\mathbf{4 b}$ (top) and $\mathbf{9}$ (bottom) (solid samples, $80 \mathrm{~K}$ ).

Table S3. Mössbauer parameters at $80 \mathrm{~K}$ for compounds $\mathbf{4 a}$ - 9. If not otherwise stated the samples were measured as solid material.

\begin{tabular}{cccc}
\hline Compound & $\boldsymbol{\delta}\left(\mathrm{mm} \mathrm{s}^{-\mathbf{1}}\right)$ & $\Delta \mathrm{E}_{\mathrm{Q}}\left(\mathbf{m m ~ s}^{-\mathbf{1}}\right)$ & area (\%) \\
\hline $\mathbf{4 a}$ & 0.73 & 2.82 & 100 \\
$\mathbf{4 b}$ & 0.69 & 3.04 & 100 \\
$\mathbf{5}$ & 0.45 & 0.11 & 100 \\
$\mathbf{6}$ & 0.41 & 0.18 & 100 \\
$\mathbf{7}$ & 0.73 & 2.96 & 93 \\
& 1.41 & 3.03 & $7^{\mathrm{a})}$ \\
$\mathbf{7}$ dissolved in $^{\text {MeCN }}{ }^{\mathrm{b})}$ & 0.93 & 3.12 & 12 \\
& 0.92 & 2.47 & 48 \\
$\mathbf{8}$ & 0.37 & 0.12 & 40 \\
& 0.37 & 0.20 & 45 \\
$\mathbf{9}$ & 0.72 & 3.19 & 55 \\
& 0.36 & 0.25 & 50 \\
\hline
\end{tabular}

a) high spin $\mathrm{Fe}^{2+}$ impurity. ${ }^{\text {b) }}$ Deviations from the solid sample of 7 and 8 can be explained due to solvent effects. 

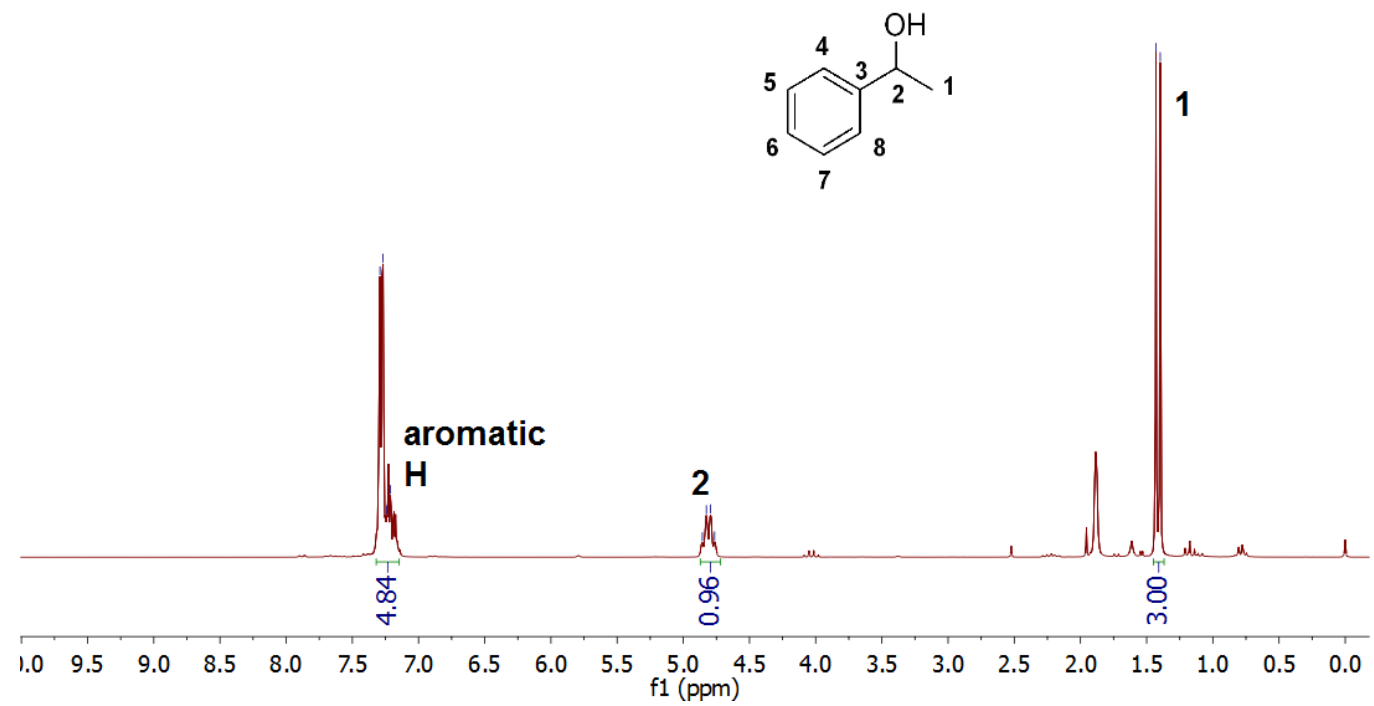

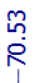

6
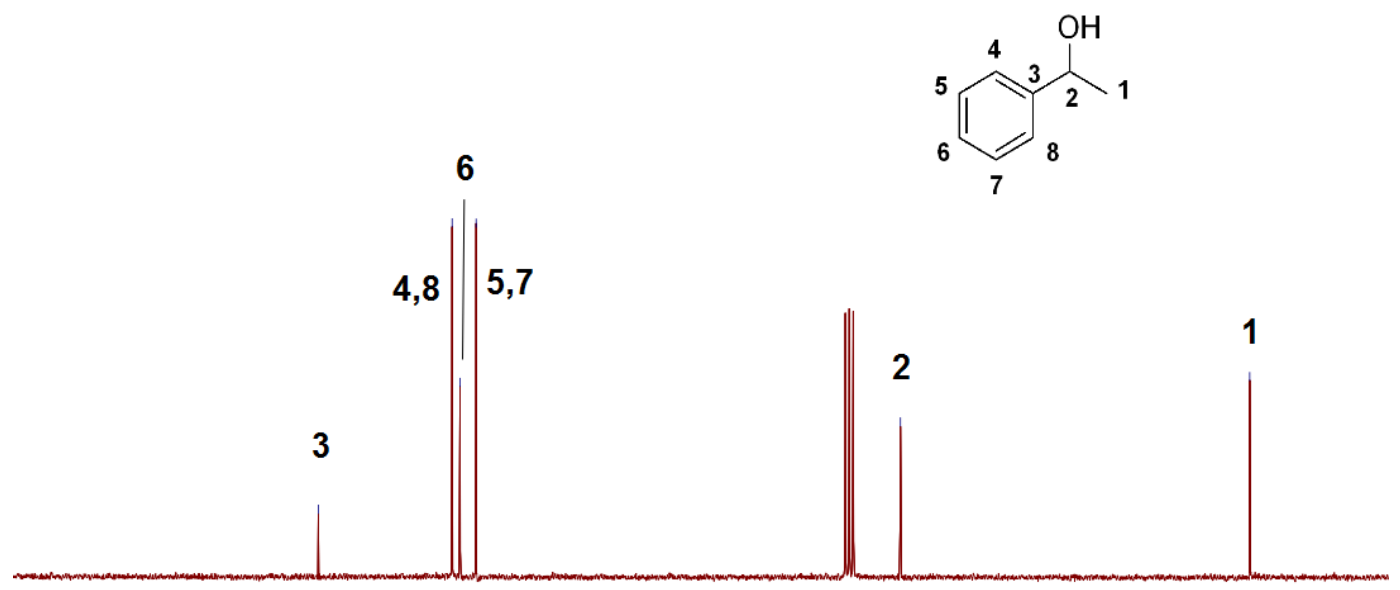

$\begin{array}{lllllllll}180 & 170 & 160 & 150 & 140 & 130 & 120 & 110 & 100\end{array}$

Figure S21. ${ }^{1} \mathrm{H}$ (top) and ${ }^{13} \mathrm{C}$ NMR spectra (bottom) of 1-phenylethan-1-ol. 

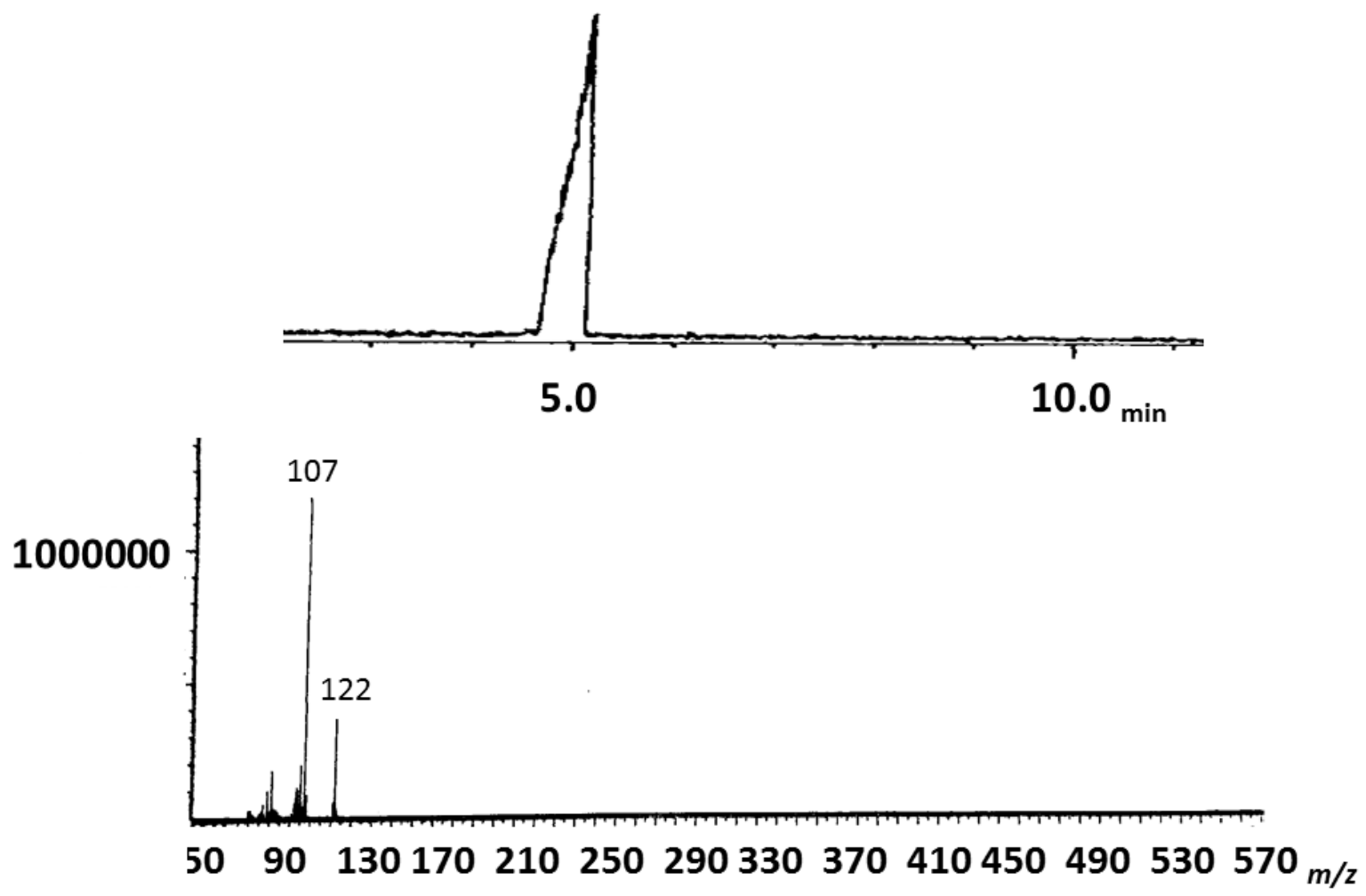

Figure S22. GC-trace (top) and MS- spectrum (bottom) of 1-phenylethan-1-ol. 
Table S4. Crystal Data and Refinement Details for the Crystal Structure Analyses of Compounds 4a, 4b, 5, 6.

\begin{tabular}{|c|c|c|c|c|}
\hline & $4 a$ & $4 b$ & 5 & 6 \\
\hline Empirical formula & $\mathrm{C}_{40} \mathrm{H}_{39} \mathrm{Cl}_{2} \mathrm{FeP}_{3} \mathrm{Si}$ & $\mathrm{C}_{40} \mathrm{H}_{39} \mathrm{Br}_{2} \mathrm{FeP}_{3} \mathrm{Si}$ & $\mathrm{C}_{50} \mathrm{H}_{56} \mathrm{FeOP}_{3} \mathrm{SiN}_{3} \mathrm{~B}_{2} \mathrm{~F}_{8}$ & $\mathrm{C}_{49} \mathrm{H}_{51} \mathrm{~B}_{2} \mathrm{~F}_{8} \mathrm{FeN}_{4} \mathrm{P}_{3}$ \\
\hline Formula weight & 767.51 & 856.38 & 1065.45 & 1018.38 \\
\hline Temperature/K & 293 & $104.7(10)$ & $173(2)$ & 293 \\
\hline Crystal system & Triclinic & Triclinic & Monoclinic & Monoclinic \\
\hline Space group & $P 1$ & $P 1$ & $P 2_{1} / C$ & $P 2_{1} / n$ \\
\hline$a / \AA$ & $9.341(7)$ & $9.4197(3)$ & $13.1059(11)$ & $13.352(7)$ \\
\hline b/Å & $9.463(7)$ & $9.4229(3)$ & $15.7016(9)$ & $18.685(10)$ \\
\hline$c / \AA$ & $12.799(9)$ & $12.7283(3)$ & $26.135(2)$ & $19.802(10)$ \\
\hline$\alpha /^{\circ}$ & $93.560(8)$ & $110.289(3)$ & 90 & 90 \\
\hline$\beta /^{\circ}$ & $109.985(7)$ & $93.258(2)$ & $102.265(4)$ & $90.679(8)$ \\
\hline$y l^{\circ}$ & $112.1366(11)$ & $112.426(3)$ & 90 & 90 \\
\hline VolumelA $\AA^{3}$ & $961.0(12)$ & $955.94(5)$ & $5255.5(7)$ & $4940(5)$ \\
\hline$\underset{\rho_{\text {calc }} \mathbf{g} / \mathrm{cm}^{3}}{Z}$ & $\begin{array}{c}1 \\
1.3262\end{array}$ & $\begin{array}{c}1 \\
1.488\end{array}$ & $\begin{array}{c}4 \\
1.347\end{array}$ & $\begin{array}{c}4 \\
1.3692\end{array}$ \\
\hline$\mu / \mathrm{mm}^{1}$ & 0.715 & 7.305 & 0.469 & 0.471 \\
\hline$F(000)$ & 399.1 & 434.0 & 2208.0 & 2107.7 \\
\hline Crystal size/mm ${ }^{3}$ & $0.42 \times 0.31 \times 0.25$ & $0.14 \times 0.11 \times 0.10$ & $0.16 \times 0.14 \times 0.11$ & $0.29 \times 0.14 \times 0.11$ \\
\hline Radiation & $\operatorname{MoK}_{\alpha}(\lambda=0.71075)$ & $\operatorname{CuK}_{\alpha}(\lambda=1.54184)$ & $\operatorname{MoK}_{\alpha}(\lambda=0.71075)$ & $\operatorname{MoK}_{\alpha}(\lambda=0.71075)$ \\
\hline 20 range for data collection $/^{\circ}$ & 3.48 to 51.38 & 7.58 to 148.42 & 3.18 to 50 & 3.7 to 55 \\
\hline Index ranges & $\begin{array}{c}-11 \leq \mathrm{h} \leq 11,-11 \leq \mathrm{k} \leq 11 \\
-15 \leq \mathrm{I} \leq 15\end{array}$ & $\begin{array}{c}-11 \leq \mathrm{h} \leq 11,-11 \leq \mathrm{k} \leq 11 \\
-15 \leq \mathrm{I} \leq 15\end{array}$ & $\begin{array}{c}-15 \leq h \leq 15,-18 \leq \mathrm{k} \leq 18 \\
-31 \leq \mathrm{I} \leq 31\end{array}$ & $\begin{array}{c}-17 \leq \mathrm{h} \leq 17,-24 \leq \mathrm{k} \leq 24 \\
-25 \leq \mathrm{I} \leq 25\end{array}$ \\
\hline Reflections collected & 8788 & 18125 & 44522 & 51614 \\
\hline Independent reflections & 7096 & 6887 & 9235 & 11327 \\
\hline Data/restraints/parameters & $7096 / 0 / 424$ & $6887 / 3 / 425$ & $9235 / 19 / 648$ & $11327 / 0 / 608$ \\
\hline${ }^{\mathrm{a}}$ Goodness-of-fit on $\mathrm{F}^{2}$ & 0.960 & 1.074 & 1.025 & 1.043 \\
\hline${ }^{b, c}$ Final $R$ indexes $[I \geq 2 \sigma(I)]$ & $\mathrm{R}_{1}=0.0326, \mathrm{wR}_{2}=0.0643$ & $\mathrm{R}_{1}=0.0195, \mathrm{wR}_{2}=0.0539$ & $\mathrm{R}_{1}=0.0195, \mathrm{wR}_{2}=0.0539$ & $\mathrm{R}_{1}=0.0526, w \mathrm{R}_{2}=0.1410$ \\
\hline Final R indexes [all data] & $\mathrm{R}_{1}=0.0354, \mathrm{wR}_{2}=0.0656$ & $\mathrm{R}_{1}=0.0354, \mathrm{wR}_{2}=0.0656$ & $\mathrm{R}_{1}=0.0195, \mathrm{wR}_{2}=0.0539$ & $\mathrm{R}_{1}=0.0691, w R_{2}=0.1570$ \\
\hline Largest diff. peak/hole / e $\AA^{-3}$ & $0.17 /-0.29$ & $0.17 /-0.29$ & $0.69 /-0.29$ & $0.72 /-0.65$ \\
\hline Flack parameter & $-0.050(10)$ & $-0.050(10)$ & - & - \\
\hline$C C D C$ reference & 1061126 & 1061127 & 1061128 & 1061129 \\
\hline
\end{tabular}


Table S5. Crystal Data and Refinement Details for the Crystal Structure Analyses of Compounds 7, 8, 9.

\begin{tabular}{|c|c|c|c|}
\hline & 7 & 8 & 9 \\
\hline $\begin{array}{l}\text { Empirical formula } \\
\text { Formula weight }\end{array}$ & $\begin{array}{c}\mathrm{C}_{41} \mathrm{H}_{39} \mathrm{Cl}_{2} \mathrm{FeP}_{3} \\
751.44\end{array}$ & $\begin{array}{c}\mathrm{C}_{51} \mathrm{H}_{54} \mathrm{Cl}_{6} \mathrm{Fe}_{3} \mathrm{~N}_{5} \mathrm{P}_{3} \\
1210.20\end{array}$ & $\begin{array}{c}\mathrm{C}_{53} \mathrm{H}_{57} \mathrm{Br}_{4} \mathrm{Fe}_{2} \mathrm{~N}_{6} \mathrm{P}_{3} \\
1302.30\end{array}$ \\
\hline Temperature/K & 293 & 293 & $104.4(14)$ \\
\hline Crystal system & Triclinic & Monoclinic & Monoclinic \\
\hline Space group & $P 1$ & $P 2_{1} / c$ & $C c$ \\
\hline$a / \bar{A}$ & $9.286(10)$ & $13.1885(10)$ & $31.1772(10)$ \\
\hline b/Å & $9.318(10)$ & $14.8199(12)$ & $13.33897(12)$ \\
\hline$c / \AA \AA A$ & $12.642(13)$ & $28.7052(19)$ & $17.8988(6)$ \\
\hline$\alpha /^{\circ}$ & $94.8155(18)$ & 90 & 90 \\
\hline$\beta /^{\circ}$ & $109.849(8)$ & $89.866(4)$ & $131.633(5)$ \\
\hline$Y l^{\circ}$ & $110.022(14)$ & 90 & 90 \\
\hline Volume/A $\AA^{3}$ & $941.6(17)$ & $5610.5(7)$ & $5563.5(3)$ \\
\hline $\mathbf{Z}$ & 1 & 4 & 4 \\
\hline$\rho_{\text {calc }} g / \mathrm{cm}^{3}$ & 1.3251 & 1.4326 & 1.555 \\
\hline$\mu / \mathrm{mm}^{1}$ & 0.698 & 1.175 & 8.677 \\
\hline$F(000)$ & 391.1 & 2489.6 & 2616.0 \\
\hline Crystal size $/ \mathrm{mm}^{3}$ & $0.21 \times 0.17 \times 0.16$ & $0.37 \times 0.24 \times 0.23$ & $0.17 \times 0.15 \times 0.11$ \\
\hline Radiation & $\operatorname{MoK}_{\alpha}(\lambda=0.71075)$ & $\operatorname{MoK}_{\alpha}(\lambda=0.71075)$ & $\operatorname{MoK}_{\alpha}(\lambda=0.71075)$ \\
\hline $2 \Theta$ range for data collection $/{ }^{\circ}$ & 3.52 to 52.82 & 3.96 to 61.16 & 7.58 to 148.24 \\
\hline Index ranges & $-11 \leq \mathrm{h} \leq 11$ & $-18 \leq h \leq 18$ & $-31 \leq h \leq 38$ \\
\hline Reflections collected & 9162 & 66759 & 19660 \\
\hline Independent reflections & 7487 & 17038 & 8932 \\
\hline Data/restraints/parameters & $7487 / 0 / 424$ & $17038 / 0 / 658$ & $8932 / 2 / 620$ \\
\hline${ }^{\mathrm{a}}$ Goodness-of-fit on $\mathrm{F}^{2}$ & 0.898 & 1.033 & 1.026 \\
\hline${ }^{b, c}$ Final $R$ indexes $[I \geq 2 \sigma(I)]$ & $\mathrm{R}_{1}=0.0374, \mathrm{wR}_{2}=0.0807$ & $\mathrm{R}_{1}=0.0444, \mathrm{wR}_{2}=0.1179$ & $\mathrm{R}_{1}=0.0333, \mathrm{wR}_{2}=0.0859$ \\
\hline Final $\mathbf{R}$ indexes [all data] & $\mathrm{R}_{1}=0.00436, \mathrm{wR}_{2}=0.0858$ & $\mathrm{R}_{1}=0.0585, \mathrm{wR}_{2}=0.1327$ & $\mathrm{R}_{1}=0.0346, \mathrm{wR}_{2}=0.0869$ \\
\hline Largest diff. peak/hole / e $A^{-3}$ & $0.33 /-0.33$ & $0.78 /-0.95$ & $0.94 /-0.60$ \\
\hline Flack parameter & $-0.022(13)$ & - & $0.001(3)$ \\
\hline CCDC reference & 1061130 & 1061131 & 1061132 \\
\hline
\end{tabular}


Table S6. Relative energy differences (in $\mathrm{kcal} / \mathrm{mol}$ ) of the calculated conformers of tetrahedral $4 \mathrm{a}$ and 7 (B3LYP/6-31G(2d,p)).

\begin{tabular}{lll}
\hline Conformation & Triphos & Triphos $^{\mathrm{Si}}$ \\
\hline & $\Delta \mathrm{E}=0.00$ & $\Delta \mathrm{E}=0.00$ \\
$\Delta \mathrm{G}=0.00$ & $\Delta \mathrm{G}=0.00$ \\
& $\mathrm{E}=-4793.494192$ hartree & $\mathrm{E}=-5044.947071$ hartree \\
& $\mathrm{G}=-4793.583629$ hartree & \\
chair, third arm equatorial & & \\
\hline
\end{tabular}

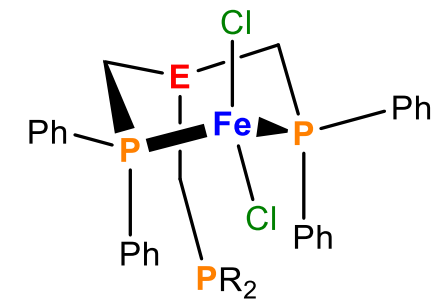
$\Delta \mathrm{E}=1.51$
$\Delta \mathrm{E}=0.22$
$\Delta \mathrm{G}=3.21$
$\Delta \mathrm{G}=1.09$

chair, third arm axial

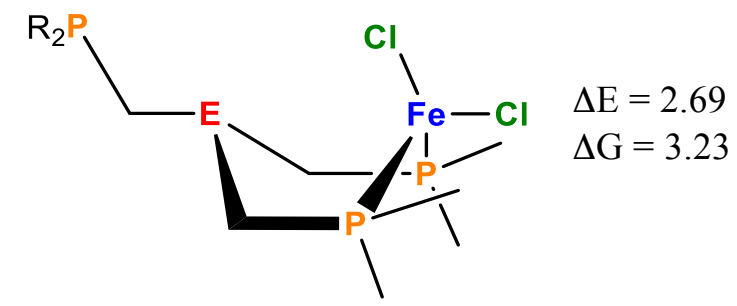

$\Delta \mathrm{E}=5.66$
$\Delta \mathrm{G}=4.62$

boat, third arm equatorial

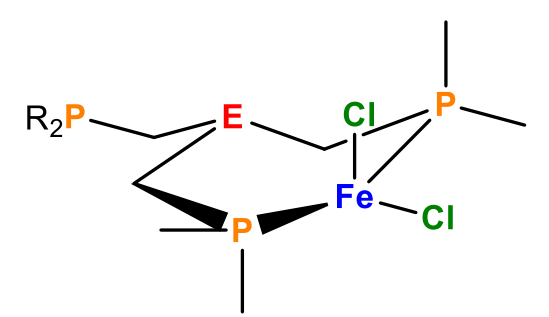

$\Delta \mathrm{E}=2.35$
$\Delta \mathrm{G}=1.87$

$\Delta \mathrm{E}=1.73$

$\Delta \mathrm{G}=1.29$

twisted, third arm equatorial<smiles>[R2]C[C@H]1C[P](C)(C)[P](C)(C)[C@H]1Cl</smiles>
$\Delta \mathrm{E}=2.17$
$\Delta \mathrm{E}=1.73$
$\Delta \mathrm{G}=2.23$
$\Delta \mathrm{G}=1.29$

twisted, third arm axial

All DFT calculations were performed using the Gaussian09 D.01 software package.(1) Input structures were prepared based on the crystal structures of $\mathbf{4 a}$ and 7 . The geometries were optimized at the B3LYP/6-31G(2d,p) level of theory 
under tight convergence criteria and an ultrafine integration grid. For some calculations, implicit solvent effects were taken into account by using the polarizable continuum model (2) (IEFPCM).

\section{NBO analysis}

NBO analysis was carried out on optimized structures of tetrahedral Triphos and Triphos ${ }^{\mathrm{Si}}$ complexes.

\section{Second order perturbation analysis:}

Interaction $\mathrm{LP}(\mathrm{P}) \cdot \mathrm{LP} *(\mathrm{Fe})-\sigma$-bonding

for Triphos:

\begin{tabular}{|c|c|c|c|c|c|c|c|c|c|c|}
\hline 189. LP & ( & 1) & P & 2 & 1185. LP* & 6) $\mathrm{Fe}$ & 1 & 35.30 & 0.41 & 0.158 \\
\hline 189. LP & ( & 1) & $\mathrm{P}$ & 2 & /186. LP* & 7) $\mathrm{Fe}$ & 1 & 2.17 & 0.47 & 0.041 \\
\hline 189. LP & ( & 1) & $\mathrm{P}$ & 2 & $1187 . \mathrm{LP}^{*}($ & 8) $\mathrm{Fe}$ & 1 & 14.08 & 0.47 & 0.105 \\
\hline 189. LP & ( & 1) & $\mathrm{P}$ & 2 & $1188 \cdot \mathrm{LP}^{*}($ & 9) $\mathrm{Fe}$ & 1 & 10.57 & 0.45 & 0.089 \\
\hline 190. LP & ( & 1) & $\mathrm{P}$ & 3 & /185. LP*( & 6) $\mathrm{Fe}$ & 1 & 35.36 & 0.41 & 0.159 \\
\hline 190. LP & ( & 1) & $\mathrm{P}$ & 3 & /186. LP* ( & 7) $\mathrm{Fe}$ & 1 & 13.62 & 0.47 & 0.103 \\
\hline 190. LP & ( & 1) & $\mathrm{P}$ & 3 & $1187 \cdot \mathrm{LP}^{*}($ & 8) $\mathrm{Fe}$ & 1 & 5.20 & 0.47 & 0.064 \\
\hline 190. LP & ( & 1) & $\mathrm{P}$ & 3 & $1188 \cdot \mathrm{LP}^{*}($ & 9) $\mathrm{Fe}$ & 1 & 6.88 & 0.45 & 0.072 \\
\hline \multicolumn{11}{|c|}{ sum $=122.41$} \\
\hline 193. LP & ( & 1) & $\mathrm{P}$ & 2 & /189. LP*( & 6) $\mathrm{Fe}$ & 1 & 35.20 & 0.40 & 0.156 \\
\hline 193. LP & ( & 1) & $\mathrm{P}$ & 2 & /190. LP* ( & 7) $\mathrm{Fe}$ & 1 & 13.03 & 0.47 & 0.101 \\
\hline 193. LP & ( & 1) & $\mathrm{P}$ & 2 & /191. LP* ( & 8) $\mathrm{Fe}$ & 1 & 2.28 & 0.46 & 0.042 \\
\hline 193. LP & ( & 1) & $\mathrm{P}$ & 2 & /192. LP* ( & 9) $\mathrm{Fe}$ & 1 & 10.13 & 0.44 & 0.086 \\
\hline 194. LP & ( & 1) & $\mathrm{P}$ & 3 & /189. LP* ( & 6) $\mathrm{Fe}$ & 1 & 34.28 & 0.39 & 0.154 \\
\hline 194. LP & ( & 1) & $\mathrm{P}$ & 3 & /190. LP* ( & 7) $\mathrm{Fe}$ & 1 & 12.29 & 0.47 & 0.098 \\
\hline 194. LP & ( & 1) & $\mathrm{P}$ & 3 & /191. LP* ( & 8) $\mathrm{Fe}$ & 1 & 7.65 & 0.46 & 0.077 \\
\hline 194. LP & ( & 1) & $\mathrm{P}$ & 3 & /192. LP* ( & 9) $\mathrm{Fe}$ & 1 & 7.55 & 0.44 & 0.075 \\
\hline
\end{tabular}

Interaction $\mathrm{LP}(\mathrm{Fe}) \cdot \mathrm{BD}^{*}(\mathrm{P}-\mathrm{C})-\pi$-backbonding

for Triphos:

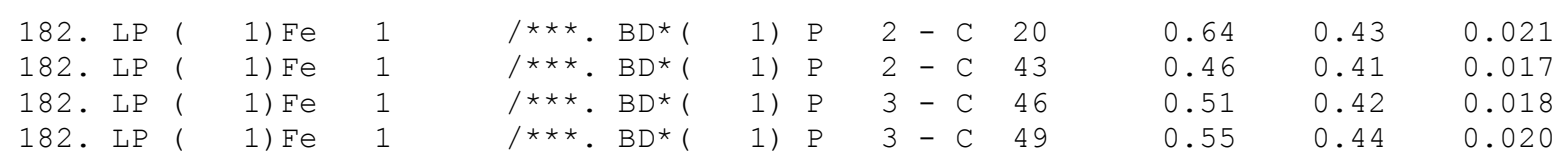

for Triphos ${ }^{\mathrm{Si}}$

\begin{tabular}{|c|c|c|c|c|c|c|c|c|c|c|c|}
\hline 186. LP ( & 1) $\mathrm{Fe}$ & 1 & $1 * * *$ & $B D *($ & 1) & $\mathrm{P}$ & 2 & $-\mathrm{C}$ & 20 & 0.71 & 0.43 \\
\hline 186. LP ( & 1) $\mathrm{Fe}$ & 1 & $1 * \star \star$ & $B D^{*}($ & 1) & P & 2 & $-\mathrm{C}$ & 43 & 0.29 & 0.42 \\
\hline 186. LP ( & 1) $\mathrm{Fe}$ & 1 & $1 * * *$ & $B D *($ & 1) & P & 3 & $-\mathrm{C}$ & 46 & 0.31 & 0.43 \\
\hline 36. LP ( & 1) $\mathrm{Fe}$ & 1 & $1 * \star \star$ & $B D *($ & 1) & P & 3 & $-\mathrm{C}$ & 49 & 0.70 & 0.44 \\
\hline
\end{tabular}




\section{Natural charges}

$\begin{array}{lll} & \text { Triphos } & \text { Triphos }^{\text {Si }} \\ \text { Fe } & 0.53683 & 0.54477 \\ \text { P(2) } & 0.98830 & 0.99812 \\ \text { P(3) } & 0.98699 & 1.00354 \\ \text { P(4) - free phosphine } & 0.84436 & 0.87039\end{array}$

Wiberg bond index

Fe-P (2)

$\mathrm{Fe}-\mathrm{P}(3)$

$\begin{array}{ll}\text { Triphos } & \text { Triphos } \\ 0.4523 & 0.4538 \\ 0.4530 & 0.4517\end{array}$

Orbital Occupancy (beta spin)

$\begin{array}{lll} & \text { Triphos } & \text { Triphos } \\ \mathrm{BD} \mathrm{Fe}-\mathrm{P}(2) & 0.96817 & 0.97247 \\ \mathrm{BD} \mathrm{Fe}-\mathrm{P}(3) & 0.97083 & 0.97024\end{array}$

\section{References}

1. M. J. Frisch, G. W. Trucks, H. B. Schlegel, G. E. Scuseria, M. A. Robb, J. R. Cheeseman, G. Scalmani, V. Barone, B. Mennucci, G. A. Petersson, H. Nakatsuji, M. Caricato, X. Li, H. P. Hratchian, A. F. Izmaylov, J. Bloino, G. Zheng, J. L. Sonnenberg, M. Hada, M. Ehara, K. Toyota, R. Fukuda, J. Hasegawa, M. Ishida, T. Nakajima, Y. Honda, O. Kitao, H. Nakai, T. Vreven, J. J. A. Montgomery, J. E. Peralta, F. Ogliaro, M. Bearpark, J. J. Heyd, E. Brothers, K. N. Kudin, V. N. Staroverov, T. Keith, R. Kobayashi, J. Normand, K. Raghavachari, A. Rendell, J. C. Burant, S. S. Iyengar, J. Tomasi, M. Cossi, N. Rega, J. M. Millam, M. Klene, J. E. Knox, J. B. Cross, V. Bakken, C. Adamo, J. Jaramillo, R. Gomperts, R. E. Stratmann, O. Yazyev, A. J. Austin, R. Cammi, C. Pomelli, J. W. Ochterski, R. L. Martin, K. Morokuma, V. G. Zakrzewski, G. A. Voth, P. Salvador, J. J. Dannenberg, S. Dapprich, A. D. Daniels, O. Farkas, J. B. Foresman, J. V. Ortiz, J. Cioslowski and D. J. Fox, Gaussian, Inc., Wallingford CT2013.

2. J. Tomasi, B. Mennucci and R. Cammi, Chem. Rev., 2005, 105, 2999-3094. 